\title{
A Probabilistic Fatigue Framework to Enable Location-Specific Lifing for Critical Thermo-mechanical Engineering Applications
}

\author{
Ritwik Bandyopadhyay ${ }^{1} \cdot$ Michael D. Sangid $^{1}$ (I)
}

Received: 5 November 2020 / Accepted: 5 January 2021 / Published online: 28 January 2021

(c) The Author(s) 2021

\begin{abstract}
The present paper describes a probabilistic framework to predict the fatigue life and failure mode under various thermomechanical loading conditions. Specifically, inclusion- and matrix-driven competing failure modes are examined within nickel-based superalloys. The critical accumulated plastic strain energy density (APSED) is employed as a unified metric to predict fatigue crack initiation in metals, which is favorable due to the usage of a single unknown parameter and its capability to predict failure across loading conditions and failure modes. In this research, we characterize the temperature-dependent variation of the critical APSED using a Bayesian inference framework and predict the competing failure modes in a coarse grain variant of RR1000 with varying strain range and temperature. The critical APSED appears to decrease along a vertically reflected sigmoidal curve with increasing temperature. Further, (a) the prediction of a failure mode, (b) failure mode associated with the minimum life, and (c) the change in the location associated with the matrix-driven failure mode with increasing temperature and decreasing strain range are consistent with the experimentally observed trends in RR1000, as well as other Nickel-based superalloys, documented in the literature. Finally, for each simulated loading condition, the uncertainty in the fatigue life is quantified as a prediction interval computed based on a 95\% confidence level of the critical APSED and the computed APSED from simulations. The overall framework provides a promising step towards microstructural-based fatigue life determination of components and enables a location-specific lifing approach.
\end{abstract}

Keywords Nickel-based superalloys $\cdot$ Inclusions $\cdot$ Crystal plasticity finite element (CPFE) - Bayesian inference · Competing failure modes $\cdot$ Location specific lifing $\cdot$ RR1000 $\cdot$ Powder metallurgy $\cdot$ Markov chain Monte Carlo

\section{Introduction}

\section{Background}

The fatigue performance of metallic materials is closely tied to their underlying microstructure, which in turn is dependent on the material processing route. Nickel (Ni)based superalloys, being an essential material for the aerospace propulsion industry, have been the focus of extensive research and development for the past several decades to seek new processing routes to achieve tailored microstructure and hence improved mechanical performance [1-3]. As an example, one may consider turbine blades. In the 1940s, wrought materials were used for such applications, whereas

Michael D. Sangid

msangid@purdue.edu

1 School of Aeronautics and Astronautics, Purdue University, West Lafayette, IN, USA later, cast materials were preferred due to their better creep performance. The turbine blade materials went through an evolution from the equiaxed to columnar (directionally solidified) to single-crystal microstructure, enabling modern turbine blades to withstand almost $60 \%$ higher temperature [1]. In turbine disks, a goal is to tailor the microstructure to achieve targeted location-specific properties and fatigue performance. Powder metallurgy (PM) processing routes, along with dual microstructure heat treatment (DMHT) strategy, are used to produce such a tailored microstructure in turbine disks $[4,5]$. DMHT processing can result in a fine grain microstructure near the disk's bore region, whereas a coarse grain microstructure is formed near the rim region. Typically, a fine grain microstructure leads to higher strength and fatigue life, whereas a coarse grain microstructure offers better creep resistance. From the DMHT turbine disk example, it is evident that a shift in the lifing methodology from classical approaches of lifing the entire component, as a monolithic structure, to new strategies of lifing the 
component at specific locations, based on the microstructure, are necessary, in order to achieve targeted location-specific performance [6]. With this in mind, the present work's objective is to predict fatigue life and associated mechanisms in a PM produced Ni-base superalloy, RR1000, at various mechanical and thermal loading conditions using a microstructure-sensitive energy-based approach. We start with a brief review of the existing literature on the microstructuresensitive fatigue life prediction and discuss the advantages of the energy-based approaches.

\section{Microstructure-Sensitive Fatigue Life Prediction}

Significant effort has been made by several researchers to incorporate microstructural variabilities in the fatigue life prediction. Notable works include fatigue indicative parameters proposed by McDowell and co-workers [7-14]; a cyclic ratcheting-based model proposed by Sinha and Ghosh [15]; effective traction-based approaches proposed by Ghosh et al. $[16,17]$; persistent slip band-based energy balance proposed by Sangid and co-workers [18-20]; energy-based approaches proposed by Dunne and colleagues [21-30], Cruzado et al. [31, 32], Bandyopadhyay et al. [33], and Prithivirajan and Sangid [34]. As we discuss below, the energy-based approaches in Refs. [21-34] are promising.

The energy-based approaches consider the fact that the motion of dislocation, i.e., plastic strain, and resistance to the motion of dislocation, i.e., shear stress, are not individually responsible for fatigue damage. It is the combination of these two local quantities, i.e., plastic work or energy, which is the driver for fatigue crack nucleation [35]. Although a significant portion of the plastic work is lost, primarily, as heat energy, the remaining portion is stored by dislocation structures and substructures. This stored energy is associated with the fatigue crack nucleation in metals. Dunne and colleagues [22-30] postulate and rationalize that the magnitude of the stored energy, as well as the length-scale over which it is stored, are collectively responsible for fatigue crack initiation. Accordingly, they have used a Griffith- or Stroh-type critical stored energy density (energy per unit area) to predict fatigue life under uniaxial [22] and multiaxial [23] loading conditions, to predict crack initiation around non-metallic inclusions [24-26], to predict crack growth within polycrystalline aggregates [27-29], and to assess fatigue performance under non-proportional loading situations [30]. Cruzado et al. [31, 32], Bandyopadhyay et al. [33], and Prithivirajan and Sangid [34] have used a volumetric critical accumulated plastic strain energy density (APSED), as opposed to energy per unit area used by Dunne et al. [22-30], to predict cycles to crack initiation. The researchers, in Refs. [31-34], have taken into account the length-scale effect implicitly via nonlocal averaging of the APSED values. In addition to the critical APSED,
Cruzado et al. [31, 32] used an additional parameter to predict fatigue life (e.g., an exponent to account for nonlinear behavior). Bandyopadhyay et al. [33] have shown that a single critical APSED value is sufficient to predict the median fatigue life and associated scatter successfully at multiple applied strain ranges and strain ratios under uniaxial loading conditions. Additionally, Prithivirajan and Sangid [34] have demonstrated that the critical APSED is well-suited to predict matrix- and pore-driven competing failure modes in additively manufactured IN718. From Refs. [23, 33], it is at least indicative, if not apparent, that the critical energy value, associated with crack initiation, for a given material is constant at a given temperature. Bandyopadhyay et al. [33] used a Bayesian framework that considers the uncertainty in the simulation and error associated with experimental fatigue life measurement to identify the critical APSED value. Thus, the energy-based fatigue life prediction approaches in Refs. [21-34], along with the calibration strategy proposed by the present authors [33], offer a path forward for validation, verification, and uncertainty quantification, which are crucial for the qualification of a new/improved material and in general, building trust in the microstructure-sensitive fatigue life prediction.

In this paper, we extend the work in [33]. First, using crystal plasticity finite element (CPFE) simulations, experimental fatigue life, and the Bayesian framework, we calibrate the critical value of APSED for RR1000 as a function of temperature. We also quantify the uncertainty in the critical APSED value originating due to the imprecise calibration of the crystal plasticity (CP) parameters and variability associated with the experimental measurement of fatigue life. Second, we use the calibrated APSED values to predict fatigue life and associated failure modes, namely inclusionand matrix-driven failures, in RR1000 as a function of strain range and temperature. Third, based on the calibrated uncertainty in the critical APSED values, we quantify uncertainty in the predicted fatigue life. Prediction of the competing failure modes, namely inclusion-driven failure (IDF) and matrix-driven failure (MDF), as a function of strain range and temperature, requires careful consideration of microstructure and defects structure that influence the emergence of these failure modes. Therefore, we briefly discuss these parameters and their implications on the competing failure modes.

\section{Parameters Influencing Competing Failure Modes in Ni-Base Superalloys}

Many researchers have experimentally investigated crack initiation from inclusions and competing failure modes in Ni-base superalloys. For example, Hyzak and Bernstein [36], and Huron and Roth [37] reported a higher percentage of IDF at a higher temperature in AF-115 and AF2-1DA, 
and René 88 DT, respectively. However, counter-evidence was also reported by Bhowal and Wusatowska-Sarnek [38] in their notched low-cycle fatigue experiments with IN718. Additionally, Hyzak and Bernstein [36] observed a change in the location of crack initiation from the surface to the interior of the specimens with decreasing strain range. Caton et al. [39], Qiu and Wu [40], and Texier et al. [41, 42] reported that surface and sub-surface inclusions were mostly associated with IDF in René 88 DT, RR1000, and IN718DA, respectively. Texier et al. [42] also noted that all surface inclusions in IN718DA were not necessarily associated with crack initiation. Caton et al. [39] observed that inclusions did not result in significant debit in life for stress amplitude greater than $1000 \mathrm{MPa}$, whereas the debit was significantly higher at lower stress amplitudes leading to a bifurcation in the stress-life plot. However, Shi et al. [43] showed counter-evidence in their research with FGH96. In Refs. [40, 41], one may notice a single isolated particle, i.e., Type 1, or an agglomerate of small particles, i.e., Type 2, can be associated with IDF. For example, Qiu and Wu [40] observed IDF from Type 1 alumina inclusions and Type 2 hafnia inclusions in RR1000, whereas Texier et al. [41] reported IDF due to Type 1 and 2 titanium nitride inclusions and Type 2 niobium carbide inclusions in IN718DA. Alexandre et al. [44] showed that particle-induced failure is more prevalent below a critical grain size of IN718. Based on an extensive experimental investigation with René 88 DT, Stinville et al. [45, 46] reported that surface crack initiation due to stress-assisted oxidation was dominant in the low-cycle fatigue regime. In the high-cycle fatigue regime, internal crack initiation, due to inclusions and crystallographic facets, was observed, leading to almost $51 \% \mathrm{MDF}$ and $49 \% \mathrm{IDF}$. They noticed failure due to crystallographic facets in the very high-cycle fatigue regime was more dominant, resulting in 95\% MDF and 5\% IDF. Zhang et al. [47-49], Naragani et al. [50], Jiang et al. [24, 51], and Chen et al. [25] carried out coupled experimental and numerical modeling work to investigate the micromechanical basis of crack initiation in RR1000 [47-50] and FGH96 [24, 25, 51] due to the presence of inclusions. From Refs. [24, 25, 47-51], we observe that significant strain-gradient, higher stored energy density, debonding of the inclusion-matrix interface, and residual stress around the inclusion are critical factors that influence slip-mediated IDF in these materials.

From the literature review, as presented above, it is evident that a multitude of parameters influences the emergence of IDF and MDF in Ni-base superalloys. We can summarize these parameters as (a) the type of the inclusion (Type 1 or 2), (b) the fracture strength of the inclusion particles, (c) the inclusion-matrix interfacial strengths, (d) the presence of a partially debonded region in the inclusionmatrix interface due to the thermo-mechanical processing of the material, (e) microstructural variability around the inclusions in terms of grain size and orientation distributions, (f) dissimilar coefficient of thermal expansion (CTE) of the inclusion and the matrix material, $(\mathrm{g})$ distance of the inclusion from the free surface, $(\mathrm{h})$ residual stress in the material due to the thermo-mechanical processing, and (i) the operating loading conditions including stress/ strain range, stress/strain ratio, and temperature. The type of the inclusion, i.e., Type 1 or 2 , determines the effective load it carries, thereby its structural stiffness. The fracture strength of the inclusion particles and the inclusion-matrix interfacial strength determine if an IDF would originate due to particle cracking or interfacial decohesion, respectively. The remaining parameters, including inclusion type, determine if crack initiation would occur near the inclusion or at a location sufficiently far from the inclusion due to slip accumulation in the matrix material. Accordingly, one would observe either an IDF or MDF, respectively.

Recently, Bandyopadhyay and Sangid [52] and Bergsmo and Dunne [26] have investigated the effects of the abovelisted parameters on the emergence of these two failure modes. Specifically, Bergsmo and Dunne [26] reported that the oxide particles' interfacial strength in RR1000 was $2050 \mathrm{MPa}$, and the fracture strength of the same particles was $2300 \mathrm{MPa}$. They showed that the particle-cracking and interfacial decohesion would be more prevalent above $1230 \mathrm{MPa}$ applied peak stress, whereas slip-induced IDFs or MDFs would be more dominant at lower stress levels. In IN718DA, Texier et al. [53] also reported particle cracking occurring at similar macroscopic stress levels (somewhere between $1147 \mathrm{MPa}$ and $1292 \mathrm{MPa}$ ) under monotonic loading. For slip-induced competing failure modes, the parametric study by Bandyopadhyay and Sangid [52] shows that none of the parameters, as mentioned above, are individually responsible for the emergence of the either of these failure modes if the inclusion size is on the order of the grain size; it is the complex interaction between these parameters, that leads to either of these failure modes. The results of [52] suggest an inherent stochastic aspect in the emergence of these failure modes, which is supported by experimental evidence in Refs. [24, 25, 36-51]. Realistic prediction of these two competing failure modes requires careful modeling of these parameters' effect in the simulation framework, as discussed in section "Polycrystalline Microstructures with Inclusions and Debonded Region".

So far, we have motivated the need for location-specific lifing (section "Background"), described the advantages of the microstructure-sensitive energy-based approaches for location-specific lifing (section "Microstructure-Sensitive Fatigue Life Prediction"), and reviewed parameters influencing the competing failure modes in Ni-base superalloys (section "Parameters Influencing Competing Failure Modesin Ni-Base Superalloys"). Now, we summarize the 
location-specific lifing framework for the present work and layout the paper's structure.

\section{Summary of the Location-Specific Lifing Framework}

For illustration, consider the schematic of a turbine disk in Fig. 1. We choose a location A in this component and demonstrate how the probability of failure plots and strainlife plots can be obtained for location A. The steps are as follows.

(i) Material characterization: Statistical descriptors of the microstructure at location A are extracted. These descriptors are grain size and orientation distributions, twin area fraction, and distribution of the inclusion size and morphology in the present context.

(ii) Virtual microstructure creation: Virtual 3D polycrystalline microstructures of the material are created. These microstructures are statistically equivalent to the microstructural attributes, characterized in the first step, and the material's strength characteristics at location $\mathrm{A}$.

(iii) Crystal plasticity simulations at a given load: $\mathrm{CPFE}$ simulations are carried out on the virtual 3D polycrystalline microstructures.

(iv) Hotspot identification and quantification of fatigue indicative metrics: In each of these microstructures, the failure location is identified, and APSED at this location is quantified.

(v) Prediction of fatigue life at the given load: Fatigue life of a given virtual 3D polycrystalline microstructure is predicted for the simulated loading condition.

(vi) Obtain probability of failure plots: Predicted fatigue lives for all virtual 3D polycrystalline microstructures for the simulated loading condition result in a probability of failure plot where each data point corresponds to a virtual 3D polycrystalline microstructure. Steps (i)-(v) can be repeated to generate the probability of failure plots for a spectrum of loading conditions.

(vii) Obtain strain-life plots: In Fig. 1, we demonstrate how to obtain the probability of failure plots using steps (i)-(v). Strain-life plots can also be generated using the same steps. For example, simulations carried out at a constant strain ratio, temperature, and varying strain ranges lead to a strain-life plot. Hence, simulations can be carried out at multiple strain ratios and temperatures to generate strain-life plots for a spectrum of loading conditions.

In section "Crystal Plasticity Finite Element Simulation Framework", steps (i)-(iii) are described in detail. The fatigue life model, i.e., steps (iv) and (v), is described in section "Fatigue Life Prediction Model". In section "Bayesian Calibration of the Critical Plastic Strain Energy Density", the Bayesian calibration framework of the fatigue life model parameter, namely critical APSED, is outlined. The results are reported and discussed in section "Results". Finally, conclusions are drawn in section "Conclusions".

\section{Crystal Plasticity Finite Element Simulation Framework}

\section{Material Characterization}

A PM produced Ni-base superalloy, RR1000, is the focus of this work. Specifically, we consider the coarse grain variant of the material, which is supersolvus heat-treated and, hence, does not contain the primary $\gamma^{\prime}$ phase. The processing of this material is described in Ref. [54]. A representative electron backscatter diffraction (EBSD) characterization of the material is shown in Fig. 2a. From the EBSD characterization, the mean grain size is $25.24 \mu \mathrm{m}$, and a random texture is observed in the material (see Fig. 2b).

Micro-computed tomography $(\mu-\mathrm{CT})$ technique is used to characterize the inclusions in this material. Specifically, inclusions in five RR1000 samples, obtained from work in Ref. [55], are characterized using $\mu$-CT. During $\mu$-CT, a $433 \mu \mathrm{m} \times 300 \mu \mathrm{m} \times 1250 \mu \mathrm{m}$ rectangular cuboid volume of the material is probed with the resolution, i.e., pixel size, of $1.5 \mu \mathrm{m}$. Afterward, Avizo software is used to process the raw data to obtain equivalent diameter and sphericity of the inclusions (see Fig. 2c, d). The sphericity [56] is computed as

sphericity $=\frac{\pi^{\frac{1}{3}}\left(6 V_{\mathrm{o}}\right)^{\frac{2}{3}}}{A_{\mathrm{o}}}$

where $A_{\mathrm{o}}$ and $V_{\mathrm{o}}$ are the surface area and volume of the inclusion, respectively, such that the sphericity represents a measure of the deviation in shape from a sphere (i.e., for a sphere, sphericity $=1$ ). The material characterization results, as shown in Fig. 2, are used to obtain 3D synthetic polycrystalline microstructures of the material, as described below.

\section{Polycrystalline Microstructures with Inclusions and Debonded Region}

A flowchart, outlining the procedure to obtain a 3D synthetic polycrystalline microstructure with an embedded inclusion, local mesh refinement, and a debonded region, is shown in Fig. 3. The process can be broadly divided into two steps: (a) creation of a 3D synthetic polycrystalline microstructure without an inclusion using statistical descriptors (grain size, 

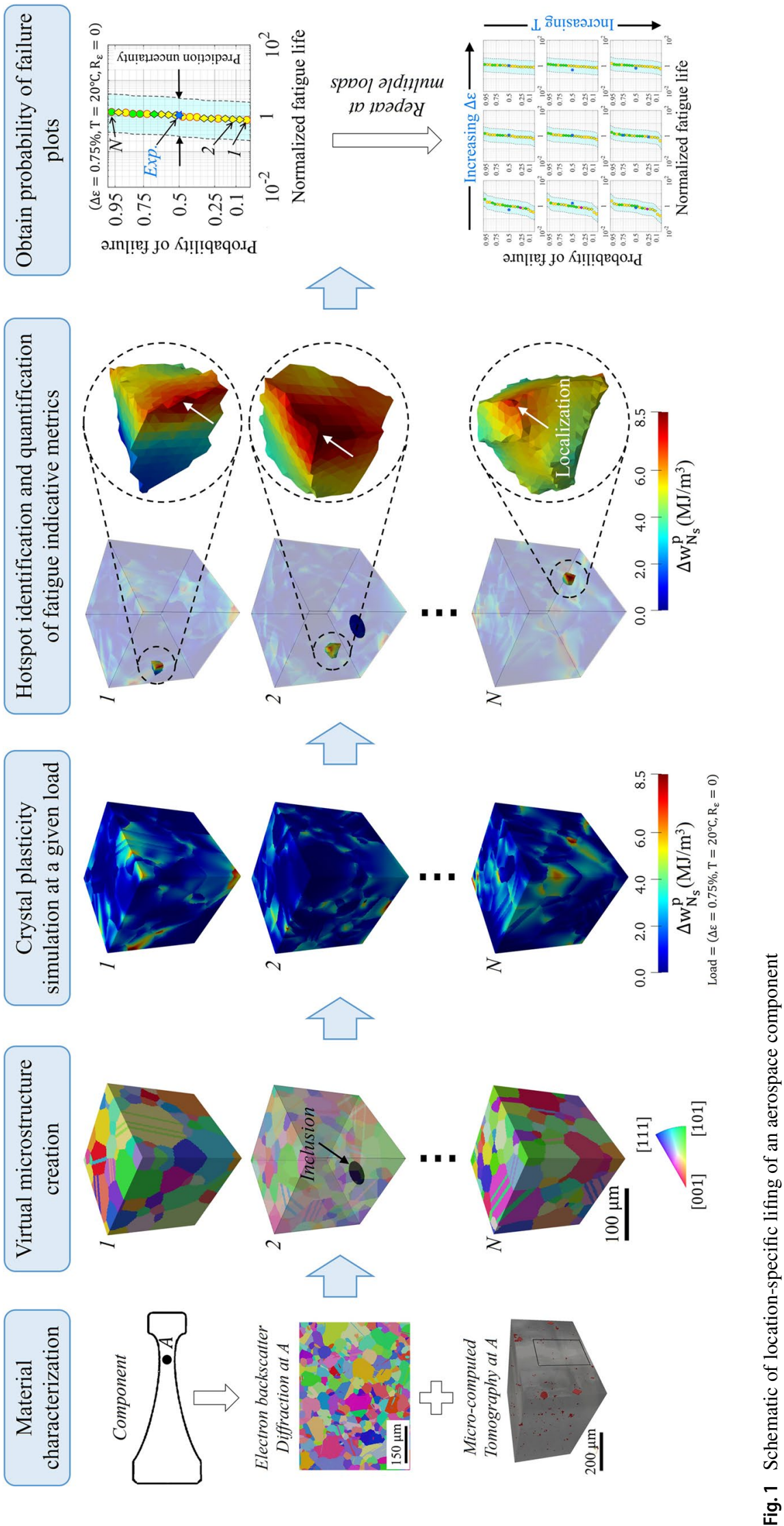

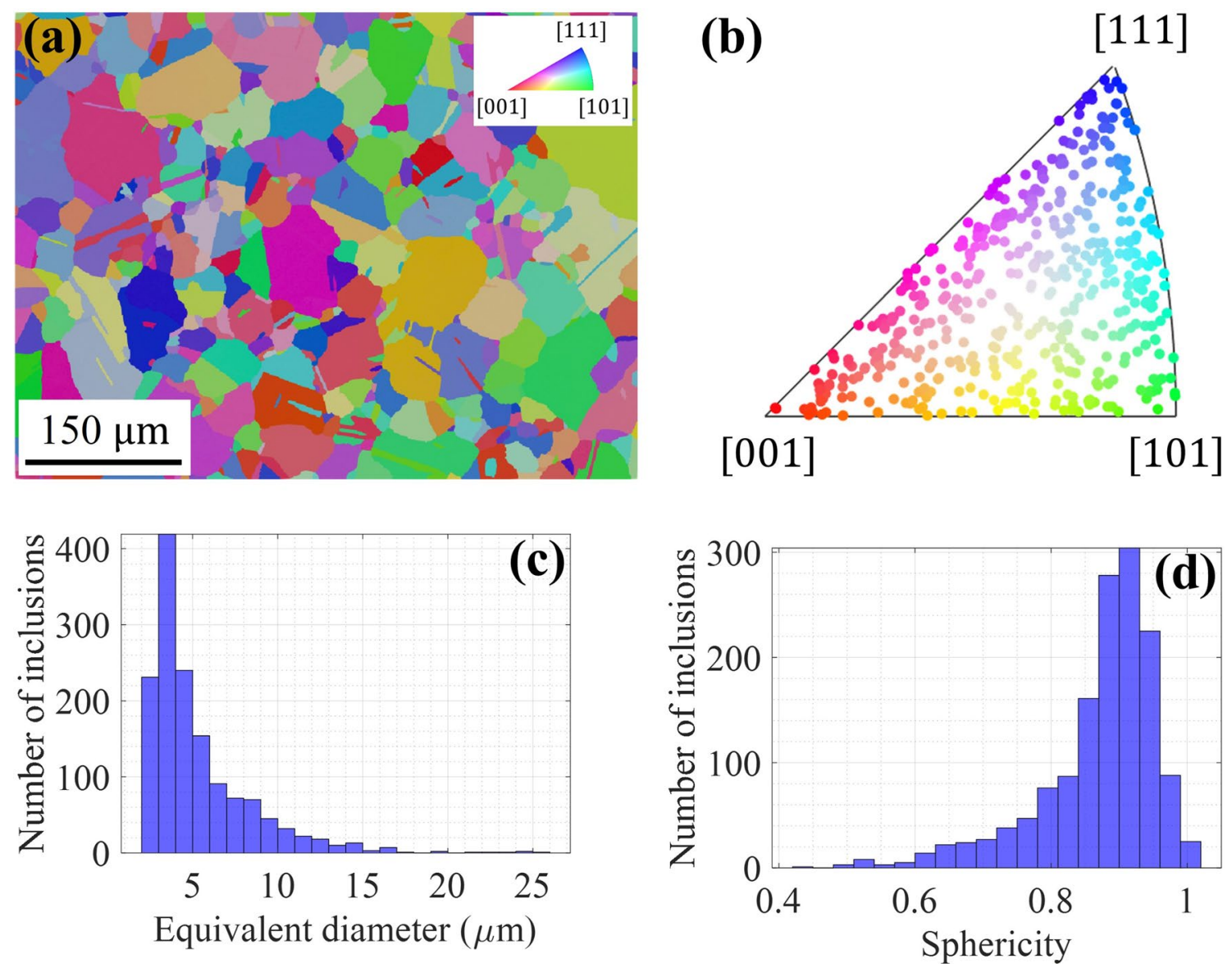

Fig. 2. a An EBSD characterization of RR1000. b The orientations of the grains in part (a) via an inverse pole figure representation confirm the random texture. Histograms of the inclusion distributions in

shape, and orientation distribution, along with the twin area fraction) of the microstructure from EBSD characterization, and (b) insertion of inclusion within these 3D synthetic polycrystalline aggregates using $\mu$-CT characterization data of the inclusion population. Each of these steps is discussed below.

Equiaxed grains are observed in RR1000. For such a case, a framework to obtain 3D synthetic polycrystalline microstructures, starting with the 2D EBSD characterization of the material, has been previously described by the present authors [20, 34, 52, 57-59]. These 3D synthetic polycrystalline microstructures are statistically equivalent to the characterized microstructural features and are sufficiently large to reproduce the microstructural strength characteristics of the material. Hence, these 3D synthetic polycrystalline microstructures are called statistically equivalent microstructures and referred to as SEMs in the remainder of this paper. Previously, Bandyopadhyay and Sangid [52] described the procedure to geometrically model an inclusion within an SEM as a solid ellipsoid. Specifically, an SEM is first discretized using finite elements (the mesh details are described in

this material, $\mathbf{c}$ equivalent diameter, and $\mathbf{d}$ sphericity obtained from the $\mu$-CT characterization of five RR1000 specimens in Ref. [55]

section "Discretization of the Statistically Equivalent Microstructures"). Subsequently, a set of elements is defined as an inclusion.

In order to predict fatigue life and associated failure mode, we need to create SEMs with realistic attributes, including (a) the number and size of the SEMs, (b) the mesh size within the discretized SEMs, and the degree of mesh refinement around the inclusion, (c) thermo-mechanical properties of the inclusion, (d) size and morphology of the inclusions, and (e) the position of the inclusions within the SEMs. Each of these items is described below.

\section{Number and Size of Statistically Equivalent Microstructures}

Ideally, a large number of SEMs is desirable to predict a stationary distribution of fatigue life. From a component design perspective, engineers are often interested in the B0.1 life or fatigue life associated with a 0.001 probability of failure [60]. The number of SEMs, necessary to obtain a stationary B0.1 life, is still an outstanding question and is not the focus of the present work. Here, we extend the framework 


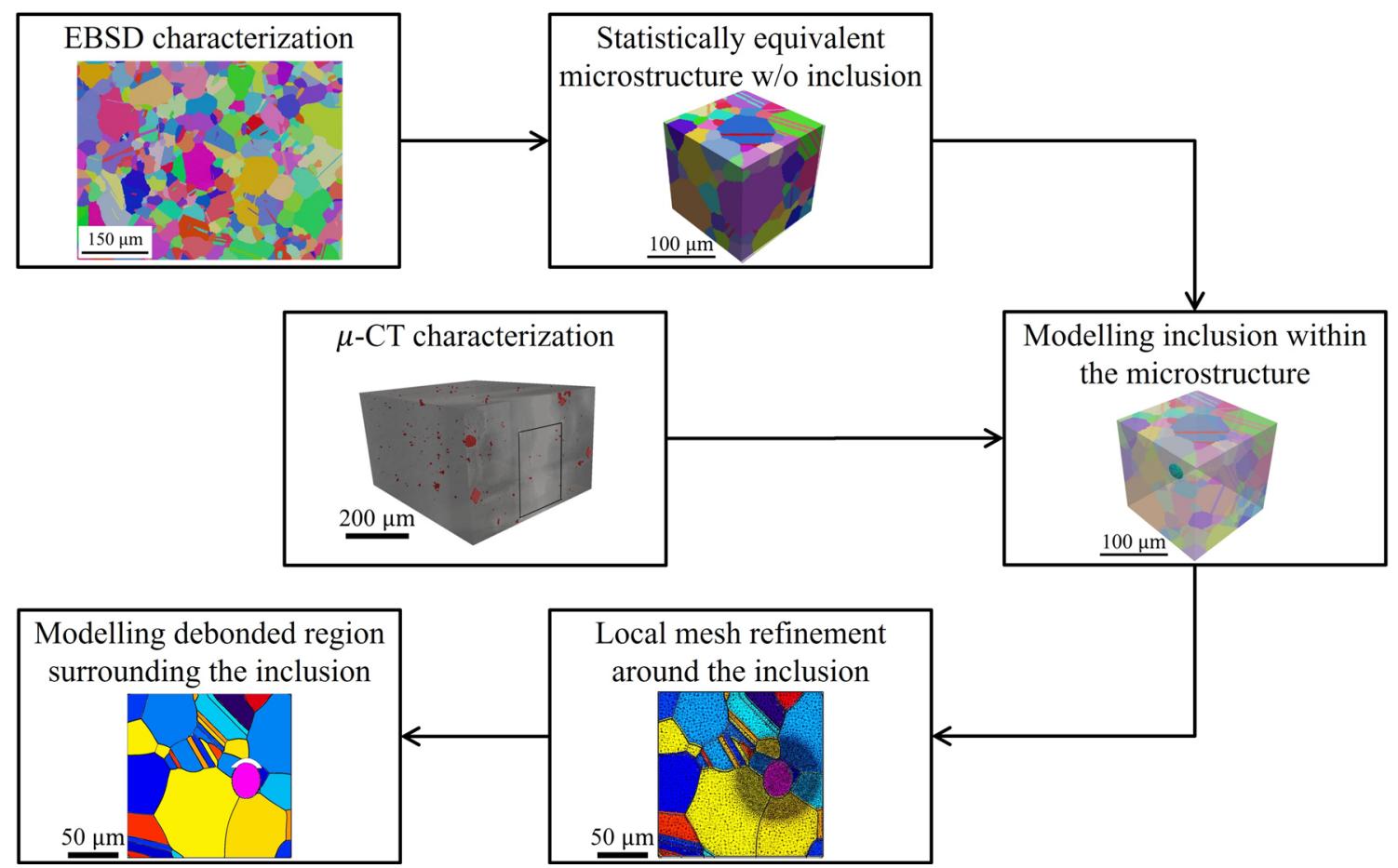

Fig. 3 A flowchart for synthetic polycrystalline microstructure creation with inclusion

described by Bandyopadhyay et al. [33] to predict the competing failure modes in RR1000 over a range of operating temperatures and applied strain range. With this, a total of 20 SEMs are created, which is consistent with previous microstructure-sensitive fatigue life predictions using SEMs in Refs. [20, 31, 33, 34]. In previous experimental research with more than 500 specimens of René 88 DT, Stinville et al. $[45,46]$ observed crack initiation from alumina and hafnia inclusions in nearly $49 \%$ of the specimens. In the present work, 10 SEMs (out of a total of 20 SEMs) are created with inclusions, with each SEM containing one inclusion. With this, a maximum of 50\% IDF can be predicted from this study.

CPFE simulations, carried out at the specimen lengthscale, would be ideal for comparative assessment between simulated and experimental fatigue life. However, such a task is impractical because of computationally expensive CPFE simulations. Recently, Prithivirajan and Sangid [34] conducted a sensitivity analysis for the SEM size compared to the full gauge volume of the microtensile fatigue specimens. Their results suggest an SEM containing $\sim 200$ grains is reasonable for fatigue life prediction. Given the grain size distribution of RR1000, cubes with dimensions of $160 \mu \mathrm{m}$ contain $\sim 200$ grains and are therefore used as SEMs in this work.

\section{Discretization of the Statistically Equivalent Microstructures}

In microstructure-sensitive fatigue life predictions, it is essential to preserve the morphology of defects, such as the inclusions, during meshing to ensure the gradients in the micromechanical field variables can be captured faithfully. Tetrahedral elements in 3D are appropriate for capturing microstructural morphologies with fewer nodes than the hexahedron elements. Hence, in this work, linear tetrahedron or C3D4 elements are chosen to discretize the SEMs.

Due to numerical issues, many researchers prefer nonlinear elements over C3D4 elements. However, such a choice is infeasible in the present work involving 240 CPFE simulations (see section "Results") with each of the SEMs having $\sim 200$ grains. Such a large number of 3D simulations with nonlinear elements is currently impractical on supercomputer clusters in a reasonable time. Moreover, many finite element solvers cannot scale down simulation time appropriately (due to non-optimal parallelization schemes) with the increase in the number of computing nodes. Hence, the choice of the C3D4 element is a trade-off between the accurate geometric representation of the polycrystalline aggregates, computational expenses, and numerical accuracy. There exist multiple ways to tackle the numerical problems that might arise due to the choice of the C3D4 elements. In this work, we adopt a regularization scheme in the post-processing phase of the analysis described in section "Nonlocal Averaging Scheme". 
Based on the mesh sensitivity study performed by Prithivirajan and Sangid [58], the element size is chosen as $\sim 3 \mu \mathrm{m}$. Further, to faithfully capture gradients in the mechanical field variables across the inclusion-matrix interfaces, the mesh is refined around the inclusion. Specifically, a spherical region with its center coinciding with the centroid of the inclusion and diameter approximately twice the equivalent diameter of the inclusion is identified and discretized using $\sim 1.2 \mu \mathrm{m} \mathrm{C3D} 4$ elements.

\section{Thermo-Mechanical Properties of Inclusions}

In this work, the inclusions are modeled as homogeneous isotropic linear elastic materials. Such an idealization is consistent with the fact that the non-metallic inclusions are brittle and often polycrystalline. Moreover, these inclusions are often pulverized into granules [61]. Hence, as explained below, we adopt a structural stiffness description of the homogenous inclusion. With this, the thermo-mechanical properties that are of interest are structural stiffness and CTE.

Depending on the chemistry (e.g., oxides, carbides) and type (Type 1 or 2), structural stiffness of the inclusions is expected to span a wide range resulting in lower or higher stiffness compared to the matrix. Here, the matrix's stiffness corresponds to Young's modulus ${ }^{1}$ of RR1000; however, the inclusion's structural stiffness is different from its Young's modulus. For example, a fully dense alumina particle has a well-defined Young's modulus value. However, depending on the type of the alumina inclusion, i.e., Type 1 or 2 , the structural stiffness, associated with the load carried by the inclusion, would be lower or higher than its Young's modulus. Prior work by the authors [52] showed that for cases when the inclusion's structural stiffness is higher than the Young's modulus of the matrix, IDFs are more likely, irrespective of the applied strain range. However, if the inclusion's structural stiffness is lower than Young's modulus of the matrix, a higher probability of IDFs is observed at a lower applied strain range compared to the higher applied strain ranges. The fatigue life dataset of RR1000 in this research shows IDF evidence for applied strain range $\leq 0.4 \%$. Hence, in this study, inclusions are predominantly modeled as structurally soft materials. However, to avoid any bias towards the stiffness value choice, the inclusion stiffness values are sampled from the shaded region of the probability distribution shown in Fig. 4a. It is to be noted

\footnotetext{
1 The reader should note that the isotropic Young's modulus of RR1000 is not used to model the matrix material. As discussed in section "Crystal Plasticity Constitutive Model", anisotropic singlecrystal elastic constants are used in the crystal plasticity modeling of the matrix material. Young's modulus of RR1000 is only referred to describe the relative structural stiffness of the inclusions.
}

that the probability distribution in Fig. 4a is hypothetical and does not represent measured structural stiffness values of inclusions in RR1000. However, the lower and upper bounds of the shaded region are motivated by the fact that the Young's modulus of porous alumina can lie somewhere between 150 and $400 \mathrm{GPa}$, depending on the pore volume fraction [62].

Due to the varying chemical composition and crystallographic structure, one may expect a wide range of CTE values for the inclusions as well, resulting in a relatively lower or higher CTE than the matrix material. In previous research with RR1000 [40] and similar PM Ni-base superalloys [45, 46], IDFs have been reported to be due to alumina and hafnia inclusions. Additionally, from chemical analysis, it has been observed that the hafnia inclusions are surrounded by alumina particles [47]. Although the CTE of bulk alumina and hafnia is lower than RR1000, depending on the monoclinic or tetragonal structure, the anisotropic CTE of hafnia [63] could be higher than the CTE of RR1000 [64]. With this, the inclusion CTEs in this work are primarily considered lower than the matrix material. However, to consider possible variabilities and avoid any bias, the CTE of the inclusions in this research is sampled from the shaded region of the probability distribution shown in Fig. 4b. The probability distribution in Fig. $4 \mathrm{~b}$ is hypothetical and does not represent the measured CTE of inclusions in RR1000. However, in the shaded region in Fig. 4b, the lower bound is based on the CTE of isotropic polycrystalline alumina and hafnia, whereas the upper bound is based on the anisotropic CTE of hafnia reported in Ref. [63].

\section{Inclusion Size and Morphology}

Based on prior work by the authors [52], the damage associated with the presence of an inclusion during cyclic loading is not directly correlated with the size and curvature of the inclusion, if the inclusion size is of the order of the mean grain size. As seen in section "Material Characterization", in the present work, the inclusion equivalent diameter is of the order of the mean grain size. To avoid any bias, the inclusion equivalent diameter and sphericity are sampled from uniform distributions in Fig. 4c, d, respectively. The lower and upper bounds of the uniform distributions in Fig. 4c, d are chosen based on the minimum and maximum values of the equivalent diameter and sphericity in Fig. 2c, d, respectively. The minimum and maximum equivalent diameters of the inclusions are found to be $2.76 \mu \mathrm{m}$ and $25.69 \mu \mathrm{m}$, respectively. The same for sphericity are 0.44 and 1 . Figure $4 \mathrm{c}, \mathrm{d}$ shows that the equivalent diameter and sphericity are sampled from the shaded regions, or in other words, the uniform distributions are truncated before sampling. Particularly, equivalent diameter values are sampled from the interval $[8,25] \mu \mathrm{m}$, and sphericity values are sampled from 

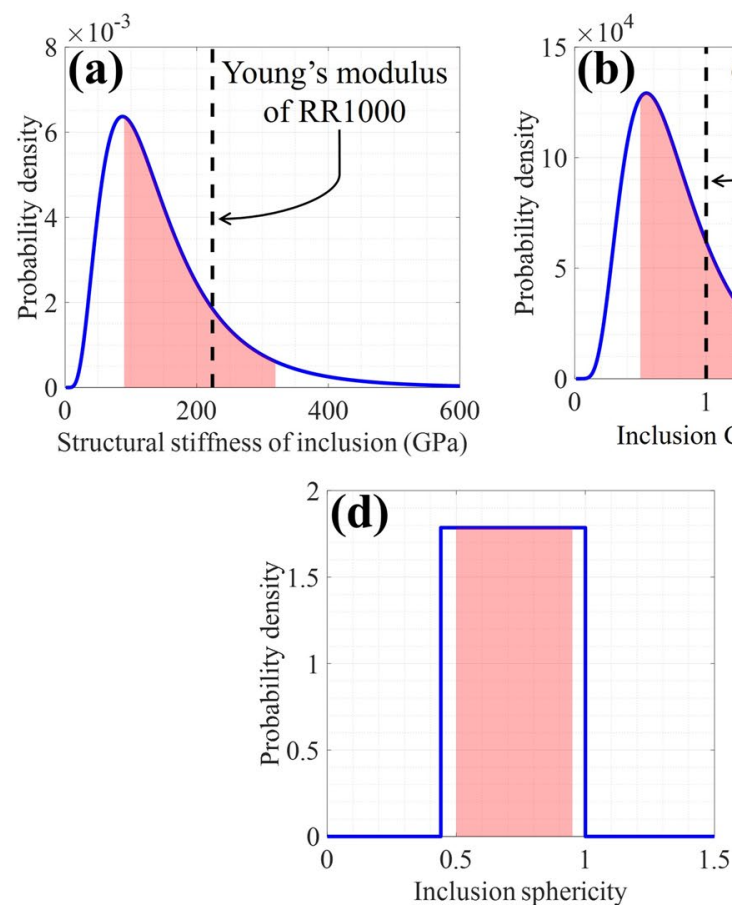

Fig. 4 Distributions of inclusion attributes for microstructure modeling. Inclusion stiffness, CTE, equivalent diameter, and sphericity values are sampled from their respective distributions' shaded regions.
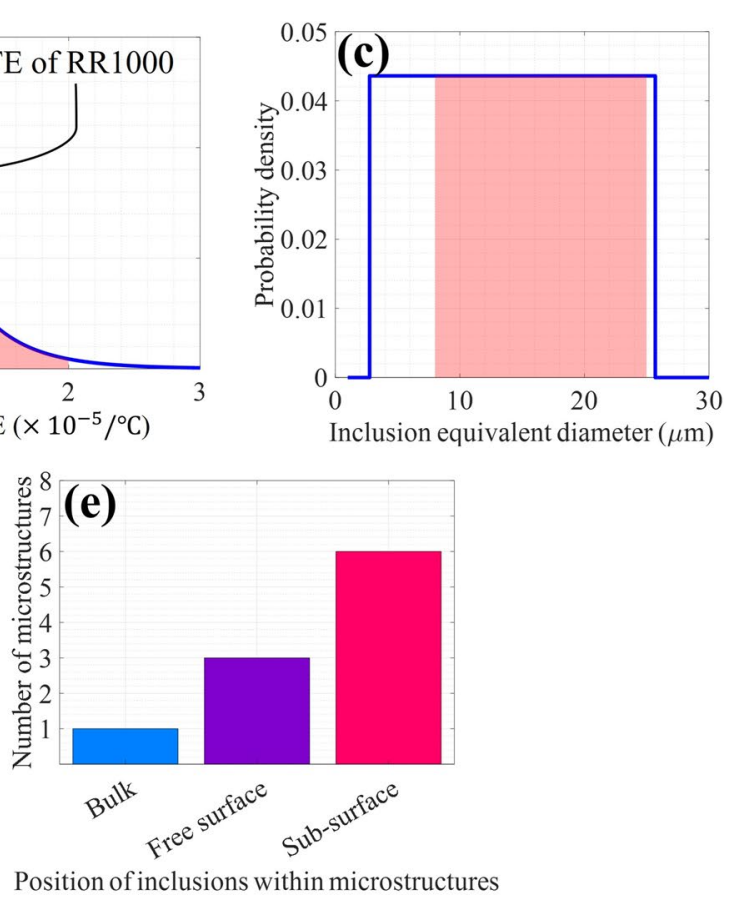

The Young's modulus and CTE values of RR1000 are indicated for reference and obtained from Refs. [64, 65] at room temperature, respectively

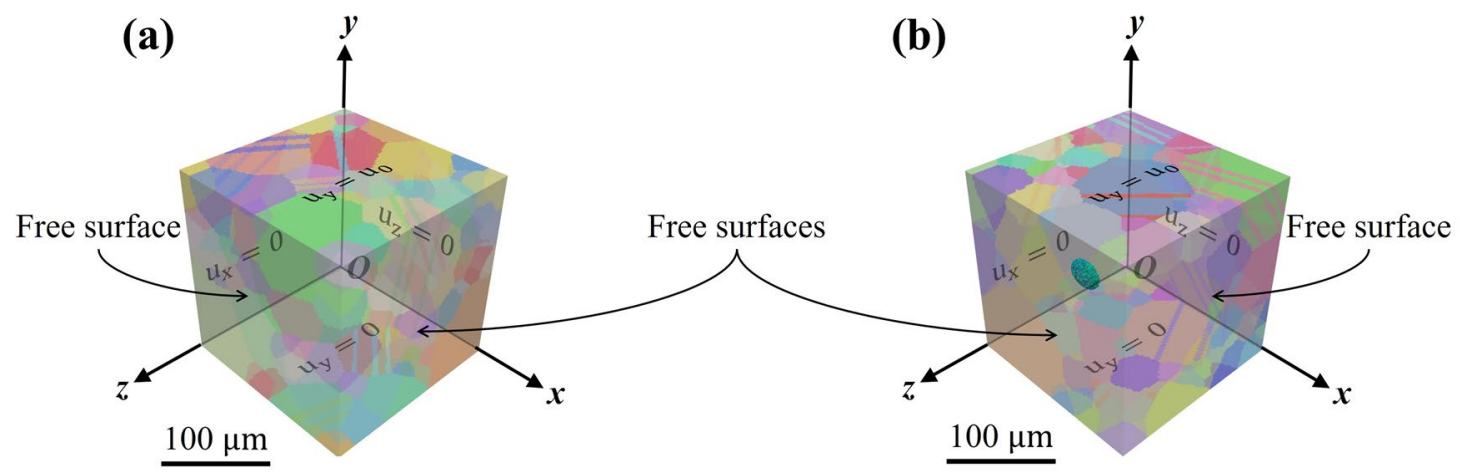

Fig. 5 Boundary conditions on a given SEM a without and $\mathbf{b}$ with an inclusion during fatigue simulation with nonzero displacement $u_{0}$ applied on the top face along the $y$-direction

the interval [0.5, 0.95]. Such a choice, especially on the lower bound of the inclusion equivalent diameter, is due to the mesh size, which is $\sim 1.2 \mu \mathrm{m}$ surrounding the inclusion.

The presence of a debonded region may not necessarily lead to an IDF [52]. However, in previous research with RR1000, IDFs have been reported to be due to the cluster of alumina and hafnia particles, i.e., Type 2 inclusions [40]. In the present experimental dataset, all IDFs originate from Type 2 inclusions, i.e., structurally soft inclusions, as well. Therefore, in this study, we introduce a partially debonded region in the inclusion-matrix interface to implicitly capture some of the effects of a Type 2 inclusion in the material, and the number of SEMs containing a partially debonded region is randomly sampled. The inclusion-matrix interface in all SEMs is assumed to be perfectly bonded, and a partially debonded region is realized by identifying and deleting a set of elements based on the study in Ref. [52]. While introducing a partially debonded region, a void of $\sim 5 \mu \mathrm{m}$ thickness is created between the inclusion and matrix (see Fig. 3), which is sufficiently large to ensure no contact 
between inclusion and matrix surfaces for up to $3 \%$ applied strain. Since, in the present research, the simulated applied strain range is $\leq 0.75 \%$, the necessity to add friction or contact surfaces between the inclusion and matrix is avoided. We note that although all inclusions in this work are geometrically modeled as ellipsoids, i.e., Type 1 inclusions, a structurally soft inclusion with a partially debonded region would implicitly capture some of the effects of a Type 2 inclusion in the material.

\section{Inclusion Position Within the Polycrystalline Aggregate}

The emergence of IDF or MDF is a consequence of complex interaction between the inclusion attributes and the microstructure surrounding the inclusion [52]. In other words, a specific location of the inclusion within the microstructure does not solely lead to a preferred failure mode. Although all surface-connected or subsurface inclusions do not lead to fatigue failure, in the present experimental dataset for RR1000, most of the IDFs originate from the surface and subsurface inclusions. Hence, in this study, the inclusions are placed at randomly chosen points on the grain boundaries, and it is ensured that inclusions in (a) three SEMs are surface-connected, (b) five SEMs are at the subsurface, and (c) one SEM is at the interior of the microstructure (see Fig. 4e). As the name suggests, the surface-connected inclusions touch one of the two free surfaces (see Fig. 5 and accompanying discussions in section "Boundary Conditions for Crystal Plasticity Finite Element Simulations)" of the SEMs. The effect of the inclusion influences the mechanical response of the surrounding anisotropic matrix material up to a spatial distance of its equivalent diameter [52] (which is indicated for each SEM based on the sampling described in section "Inclusion Size and Morphology"). Hence, the subsurface inclusions are placed within a distance of the equivalent diameter of the inclusion, $d_{\text {eq }}$, from the free surface, whereas the interior inclusions are placed at a distance greater than $d_{\mathrm{eq}}$ from the free surface.

\section{Crystal Plasticity Constitutive Model}

As mentioned before, in this work, the inclusions are modeled as homogeneous isotropic linear elastic materials, and crystal plasticity constitutive equations are adopted for the matrix material. Such an idealization is reasonable because the non-metallic inclusions are brittle, whereas plasticity is the consequence of dislocation motion in the matrix. At each integration point within a grain, the matrix material is homogenized to capture both the $\gamma$ phase and $\gamma^{\prime}$ precipitates (secondary and tertiary). A rate-dependent crystal plasticity framework, incorporating the temperature effect, is adopted to model such a homogenized response at the integration points. With this, we decompose the total deformation gradient $\mathbf{F}$ at a material point into a plastic, thermal, and elastic parts as follows:

$\mathbf{F}=\mathbf{F}^{\mathrm{e}} \mathbf{F}^{\theta} \mathbf{F}^{\mathbf{p}}$

Here, $\mathbf{F}^{\mathbf{e}}$ is associated with elastic stretch and rotation of the crystal, $\mathbf{F}^{\theta}$ is associated with the thermal expansion, and $\mathbf{F}^{\mathbf{p}}$ captures the plastic deformation. Moreover, $\mathbf{F}^{\theta}$ does not evolve with deformation, and is written as

$\mathbf{F}^{\theta}=(1+\alpha \Delta T) \mathbf{I}$

where $\mathbf{I}$ is the identity tensor, $\alpha$ is the CTE, and $\Delta T$ is the temperature change. The plastic velocity gradient, $\mathbf{L}^{\mathbf{p}}$, is written as [66]

$\mathbf{L}^{\mathbf{p}}=\dot{\mathbf{F}}^{\mathbf{p}}\left(\mathbf{F}^{\mathbf{p}}\right)^{-1}=\sum_{j=1}^{N_{\mathrm{s}}} \dot{\gamma}^{j}\left(\widehat{s}^{j} \otimes \widehat{n}^{j}\right)$

where, $\dot{\gamma}^{j}, \widehat{s}^{j}$, and $\widehat{n}^{j}$ are the shear strain rate, a unit vector along slip direction, and a unit vector along the slip plane normal associated with the $j$ th slip system, respectively; $N_{\mathrm{s}}$ is the number of slip systems, which corresponds to twelve $\langle 110\rangle\{111\}$ octahedral slip systems in RR1000. The shear strain rate $\left(\dot{\gamma}^{j}\right)$ and the resolved shear stress $\left(\tau^{j}\right)$ on the $j$ th slip system is related via a power-law flow rule [67] as follows:

$\dot{\gamma}^{j}=\dot{\gamma}_{0}\left|\frac{\tau^{j}-\chi^{j}}{g^{j}}\right|^{\frac{1}{m}} \operatorname{sgn}\left(\tau^{j}-\chi^{j}\right)$

where $\dot{\gamma}_{0}$ is the reference shearing rate, $g^{j}$ is the reference shear stress or slip system resistance, $\chi^{j}$ is the back stress, and $m$ is the strain-rate sensitivity exponent. In this work, $g^{j}$ is described via a Taylor-type hardening law

$g^{j}=g_{0}+b \mu \sqrt{h_{n} \rho^{j}}$

where $g_{0}$ is the initial slip resistance, $\rho^{j}$ is the total dislocation density on the $j$ th slip system, $b$ is the Burger's vector, $\mu$ is the shear modulus, and $h_{n}$ is a scaling parameter. We adopt a Kocks-Mecking's approach [68-70] to describe the evolution of the total dislocation density $\rho^{j}$ as

$\frac{\partial \rho^{j}}{\partial \gamma^{j}}=k_{1} \sqrt{\rho^{j}}-k_{2}(\dot{\epsilon}, T) \rho^{j}$

Here, $k_{1}$ and $k_{2}(\dot{\epsilon}, T)$ are material constants and correspond to the dislocation storage and annihilation rates, respectively; $T$ is temperature, and $\dot{\epsilon}$ is the applied strain rate. Further, the constants $k_{1}$ and $k_{2}(\dot{\epsilon}, T)$ can be explicitly related to temperature and strain-rate $[71,72]$ as follows: 
(a)

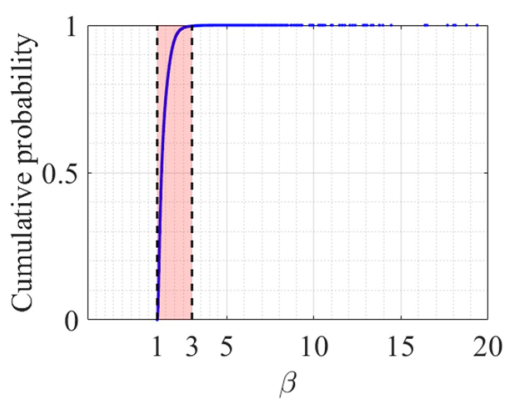

(c)

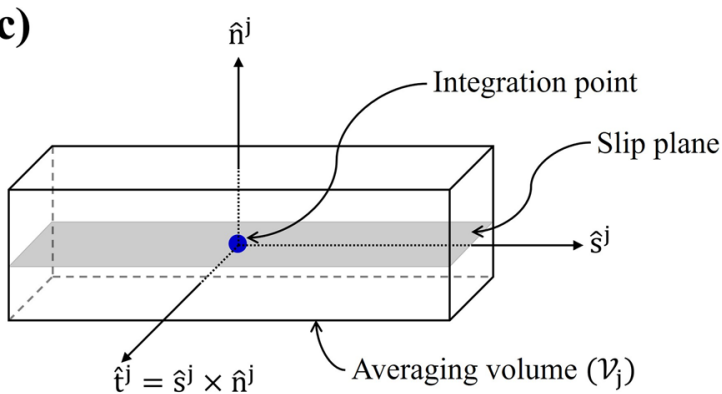

(b)

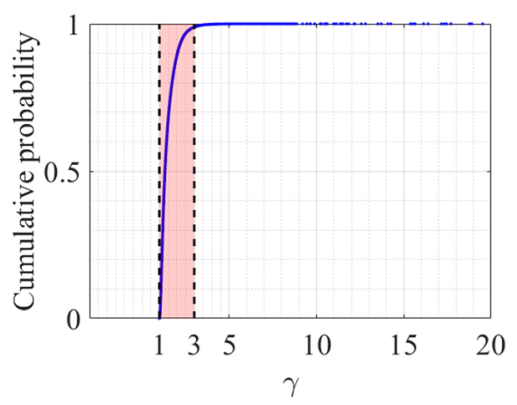

(d)

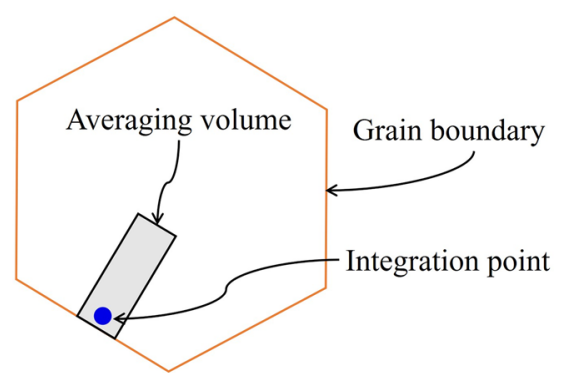

arbitrary integration point. d The averaging volume for an integration point near the grain boundary is not allowed to cross the grain boundary

$\frac{C_{44}(T)-C_{44}\left(T_{0}\right)}{C_{44}\left(T_{0}\right)}=-p_{3}\left(\frac{T-T_{0}}{T_{0}}\right)$

where $p_{1}, p_{2}$, and $p_{3}$ are positive and non-dimensional constants, and ascertained by regressions through experimentally determined Young's modulus values as a function temperature; $T_{0}$ is a reference temperature and set to $20^{\circ} \mathrm{C}$. The elastic constants at the reference temperature, i.e., $C_{11}\left(T_{0}\right), C_{12}\left(T_{0}\right)$, and $C_{44}\left(T_{0}\right)$, are adopted from Ref. [48]. The remaining CP parameters are directly used from the previous work in Ref. [52].

\section{Boundary Conditions for Crystal Plasticity Finite Element Simulations}

As indicated in Fig. 5, during CPFE simulations, the normal displacement is set to zero on three mutually orthogonal and adjacent surfaces of the SEMs, and a nonzero normal displacement is specified on one of the remaining three faces, which is orthogonal to the $y$-axis. Such a choice of the boundary conditions ensures only the $\sigma_{y y}$ component of the Cauchy stress tensor $\sigma$ to be dominant when $\sigma$ is homogenized over the entire simulation domain. Therefore, nominally, the stress state within the simulation domain is uniaxial, which would allow us to compare the simulated fatigue life data to experimental fatigue life obtained from uniaxial tests (see section "Results"). Additionally, two surfaces of the SEMs are unconstrained via the choice of 
boundary conditions and therefore treated as free surfaces of the SEMs. The presence of the free surfaces allows us to model surface-connected, subsurface, and interior inclusions and identify failures originating from the free surfaces.

\section{Residual Stress}

The effect of residual stress on the fatigue performance of metallic materials is well-recognized [76]. Since nonmetallic inclusions and matrix material in Ni-base superalloys have dissimilar CTEs, residual stress is induced in the material during the thermo-mechanical processing. Previously, Naragani et al. [50] have quantified the residual stress distribution in the vicinity of a large-seeded inclusion within RR1000 using high energy X-ray diffraction microscopy (HEDM) experiments. A temperature of $760^{\circ} \mathrm{C}$ (which is a common aging temperature [54]) was selected for the stress-free condition or $\Delta T=-740^{\circ} \mathrm{C}$ in Eq. (3), which produces a type 2 residual stress distribution similar to the HEDM measurements in Ref. [50]. Therefore, in this work, we carry out an elastic cooling simulation considering $\Delta T=-740^{\circ} \mathrm{C}$ in Eq. (3) to initialize the residual stresses in the SEMs containing inclusion. Subsequently, fatigue load is applied to the SEMs utilizing the full crystal plasticity simulations.

\section{Nonlocal Averaging Scheme}

The micromechanical field outputs from the CPFE simulations often show spurious numerical oscillations [58, 77]. Such a trend can be related to one or a combination of the following: (a) volumetric locking or shear locking due to the choice of the linear tetrahedron (C3D4) elements [77], (b) lack of mesh refinement near the grain boundaries across that may result in high gradients in field variables, (c) the effect of the boundary conditions on the elements near the corresponding surfaces, and (d) poor quality of C3D4 elements, especially near the grain boundaries [34]. To further explore the last point, i.e., the quality of the C3D4 elements, we consider two metrics, namely $\beta$ and $\gamma$, where

$\beta=\frac{C R}{3 \times I R}$

and

$\gamma=\frac{S_{\mathrm{rms}}^{3}}{8.48} \times V$

In Eq. (13), $C R$ and $I R$ represent the radii of the circumscribed and inscribed spheres to a tetrahedron element. In Eq. (14), $S_{\text {rms }}$ represents the root-mean-square value of the element's edge lengths, and $V$ is the element's volume. A
C3D4 element is good if $\beta \in[1,3]$ and $\gamma \in[1,3]$ [78]. For a randomly chosen SEM, cumulative probability plots for $\beta$ and $\gamma$ are shown in Fig. 6a, b, respectively. Most of the elements $(\sim 99 \%)$ are good and fall within the shaded region in Fig. $6 a$, b. However, $1 \%$ of the elements are poor, which might lead to spurious results from the CPFE simulations. In the literature, multiple strategies exist to address this situation. For example, Cheng et al. [77] explored three methods that are implemented during the finite element simulation to mitigate this issue, namely node-based uniform strain method, B-bar-based element method, and F-bar-based element methods. The use of an appropriate regularization scheme during the post-processing phase presents another opportunity to address implications due to poor elements. Here, we choose the second route, which also involves a significantly lower overall computation cost than the previously mentioned methods.

In recent work, Prithivirajan and Sangid have made a comparative assessment of three different averaging schemes, namely (a) nonlocal averaging, (b) band-averaging, and (c) grain-averaging [34]. Based on their analysis, it is apparent that nonlocal averaging is appropriate to predict the location of fatigue failure, fatigue life, and associated failure mode. Hence, we adopt the nonlocal averaging scheme in this work. Such an averaging scheme has previously been rationalized and used by other researchers (see, for example, [33, 52, 58, 79-82]). To illustrate the nonlocal averaging scheme, we consider an integration point as shown in Fig. 6c and identify three mutually orthogonal unit vectors passing through the integration point, namely the slip direction $\left(\widehat{s}^{j}\right)$, slip plane normal $\left(\widehat{n}^{j}\right)$, and a transverse vector $\left(\hat{t}^{\prime}\right)$ on the slip plane corresponding to the $j$ th slip system, such that these directions form a right-hand coordinate system at the integration point.

Now, a small rectangular cuboid of volume $V_{j}$ is considered having its center coinciding with the integration point and faces parallel to the three orthogonal directions defined in Fig. 6c. The micromechanical field variable is averaged over all the elements whose centroids lie within the averaging volume. One can construct twelve such volumes around the same integration point corresponding to each of the twelve octahedral slip systems, i.e., for $j=1$ to 12 . After performing averaging for all twelve such volumes, the maximum (by magnitude) of the averaged values is assigned as the slip system averaged number to that integration point.

To illustrate the nonlocal averaging scheme mathematically, let $\phi_{\mathrm{CPFE}}(\mathbf{x})$ and $\phi_{\mathrm{NLA}}(\mathbf{x})$ be the solution of a micromechanical field variable $\phi(\mathbf{x})$ from the CPFE simulation and a nonlocally averaged value of the CPFE solution, respectively. Hence, $\phi_{\mathrm{NLA}}(\mathbf{x})$ is related to $\phi_{\mathrm{CPFE}}(\mathbf{x})$ as 


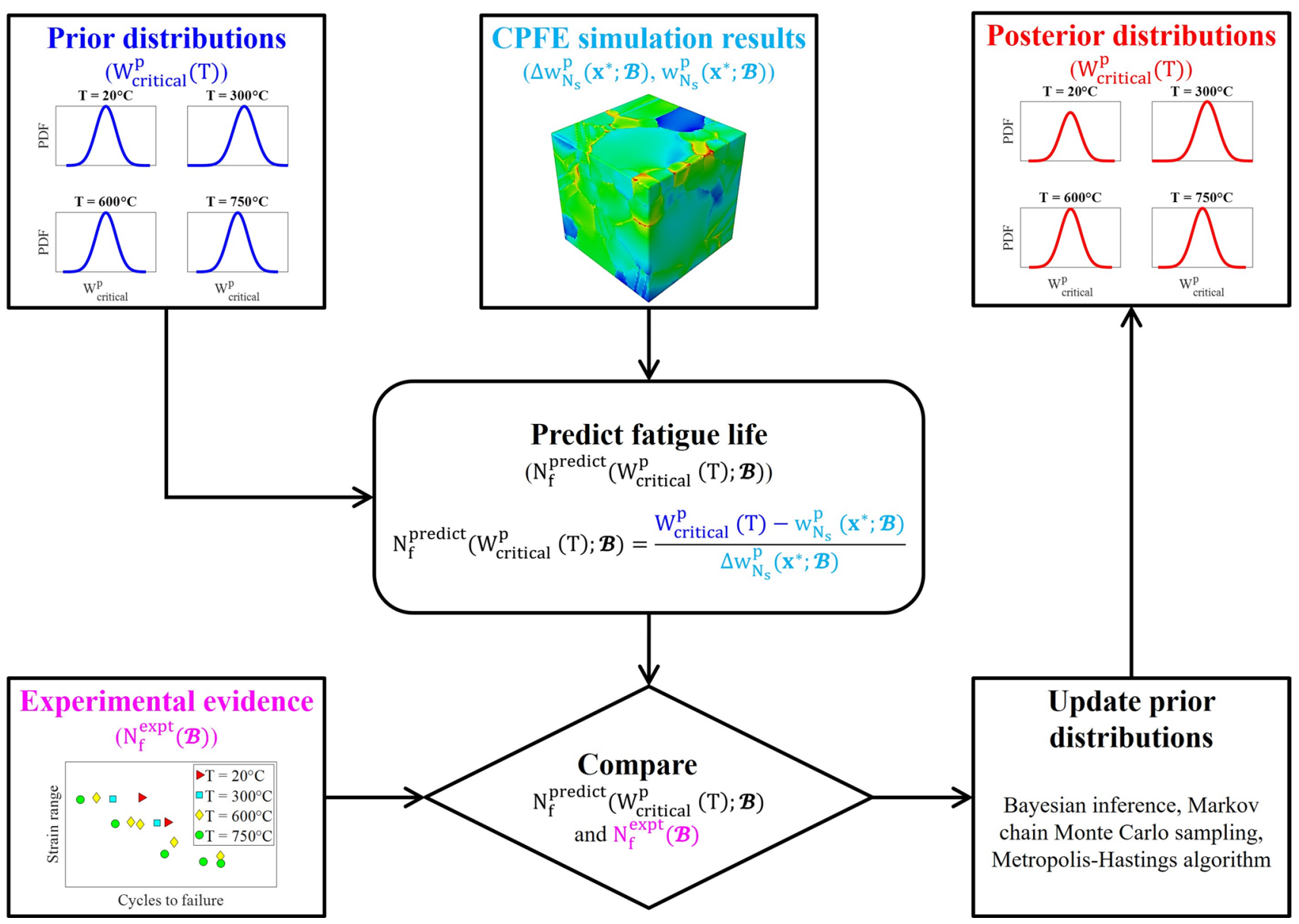

Fig. 7 Bayesian calibration of $W_{\text {critical }}^{p}(T)$ as a function of temperature

$\phi_{\mathrm{NLA}}(\mathbf{x})=\max _{j=1 \text { to } 12}\left|\frac{1}{V_{j}} \int_{V_{j}} \phi_{\mathrm{CPFE}}(\mathbf{x}) \mathrm{d} V\right|$

For integration points near the grain boundaries, the averaging volume is not allowed to cross the grain boundary (see Fig. 6d) to preserve the gradient, which is often expected across the grain boundary. The size of the averaging volume is dependent on the size of the finite elements. Based on a sensitivity analysis, the lengths of the averaging volume are defined by the size of 5, 3, 3 elements along the slip direction $\left(\hat{s}^{j}\right)$, slip plane normal $\left(\hat{n}^{j}\right)$, and transverse direction $\left(\hat{t}^{j}\right)$, respectively.

\section{Fatigue Life Prediction Model}

From the CPFE analysis, the increment of plastic strain energy density at a material point $\mathbf{x}$ over the $i$ th cycle, $\Delta w_{i}^{p}(\mathbf{x} ; \mathcal{B})$, can be written as
$\Delta w_{i}^{p}(\mathbf{x} ; \mathcal{B})=\oint_{i \text { thcycle }}\left(\sum_{j=1}^{12}\left|\tau^{j}(\mathbf{x}, t ; \mathcal{B}) \dot{\gamma}^{j}(\mathbf{x}, t ; \mathcal{B})\right|\right) \mathrm{d} t$

where $\mathcal{B}$ is a set of parameters that define loading condition, such as strain range $(\Delta \varepsilon)$, strain ratio $\left(R_{\varepsilon}\right)$, and temperature $(T)$. Accordingly, the APSED after the $i$ th cycle at the same point, $w_{i}^{p}(\mathbf{x} ; \mathcal{B})$, is expressed as

$w_{i}^{p}(\mathbf{x} ; \mathcal{B})=\sum_{k=1}^{i} \Delta w_{k}^{p}(\mathbf{x} ; \mathcal{B})$

From classical fatigue experiments, the hysteresis behavior saturates upon cyclic loading when stable dislocation structures and substructures are formed within the material [76]. If cycle $N_{\mathrm{s}}(\boldsymbol{B})$ is associated with the onset of saturation at the loading condition $\mathcal{B}$, subsequent accumulation of plastic strain energy density can be considered to be steady, at the rate of $\Delta w_{N_{\mathrm{s}}}^{p}(\mathbf{x} ; \mathcal{B})$ per cycle. Therefore, at the time of failure, i.e., after cycle $N_{\mathrm{f}}(\boldsymbol{\beta})$, one can write 
$w_{N_{\mathrm{f}}}^{p}(\mathbf{x} ; \mathcal{B})=w_{N_{\mathrm{s}}}^{p}(\mathbf{x} ; \mathcal{B})+\left(N_{\mathrm{f}}(\mathcal{B})-N_{\mathrm{s}}(\mathcal{B})\right) \cdot \Delta w_{N_{\mathrm{s}}}^{p}(\mathbf{x} ; \mathcal{B})$

Bandyopadhyay et al. [33] showed that if the material fails due to fatigue at $\mathbf{x}=\mathbf{x}^{*}, w_{N_{\mathrm{f}}}^{p}\left(\mathbf{x}^{*} ; \mathcal{B}\right)$ is a material property and independent of $\Delta \varepsilon$ and $R_{\varepsilon}$ at room temperature. They referred to $w_{N_{\mathrm{f}}}^{p}\left(\mathbf{x}^{*} ; \mathcal{B}\right)$ as the critical APSED of the material and denoted as $W_{\text {critical }}^{p}$. In this work, we hypothesize that the critical APSED, just like other material properties, such as the stiffness and strength properties of a material, is a function of temperature, i.e., $W_{\text {critical }}^{p}=W_{\text {critical }}^{p}(T)$. One objective of the present work is to ascertain the temperature-dependent variation of $W_{\text {critical }}^{p}(T)$. Subsequently, $W_{\text {critical }}^{p}(T)$ can be used to predict fatigue life using a slightly rearranged version of Eq. (18) as

$$
N_{\mathrm{f}}^{\text {predict }}\left(W_{\text {critical }}^{p}(T) ; \mathcal{B}\right)=\frac{W_{\text {critical }}^{p}(T)-w_{N_{\mathrm{s}}}^{p}\left(\mathbf{x}^{*} ; \mathcal{B}\right)}{\Delta w_{N_{\mathrm{s}}}^{p}\left(\mathbf{x}^{*} ; \mathcal{B}\right)}+N_{\mathrm{s}}(\mathcal{B})
$$

\section{Bayesian Calibration of the Critical Plastic Strain Energy Density}

If $N_{\mathrm{f}}^{\text {expt }}(\mathcal{B})$ is the experimentally obtained fatigue life at the loading condition $\mathcal{B}$, one can compute $w_{N_{\mathrm{f}}}^{p}\left(\mathbf{x}^{*} ; \mathcal{B}\right)$ using Eq. (18) as

$$
w_{N_{\mathrm{f}}}^{p}\left(\mathbf{x}^{*} ; \mathcal{B}\right)=w_{N_{\mathrm{s}}}^{p}\left(\mathbf{x}^{*} ; \mathcal{B}\right)+\left(N_{\mathrm{f}}^{\text {expt }}(\mathcal{B})-N_{\mathrm{s}}(\mathcal{B})\right) \cdot \Delta w_{N_{\mathrm{s}}}^{p}\left(\mathbf{x}^{*} ; \mathcal{B}\right)
$$

where $w_{N_{\mathrm{s}}}^{p}\left(\mathbf{x}^{*}, \beta\right)$ and $\Delta w_{N_{\mathrm{s}}}^{p}\left(\mathbf{x}^{*}, \beta\right)$ can be obtained from the CPFE simulations. However, by computing $w_{N_{\mathrm{f}}}^{p}\left(\mathbf{x}^{*}, \beta\right)$ using Eq. (20), one would not be able to obtain a single value of $W_{\text {critical }}^{p}(T)$ at a given temperature, $T$, because (a) the quantities obtained from the CPFE simulations are inherently dependent on the choice of the SEM and thereby, variability is unavoidable and (b) when experiments are repeated at a given loading condition, the fatigue life, i.e., $N_{\mathrm{f}}^{\text {expt }}(\beta)$, is never unique. Under such a situation, Bandyopadhyay et al. [33] constructed a Bayesian inference-based framework that takes into account the variabilities associated with the simulated quantities and experimental fatigue life and produces a distribution of $W_{\text {critical }}^{p}(T)$ at a given temperature, $T$. Subsequently, the mean or expected value of the distribution can be treated as a representative value of the quantity and used in Eq. (19) to predict fatigue life at temperature $T$. In Ref. [33], the experimental fatigue life data was used for a given $\Delta \varepsilon$ and $R_{\varepsilon}$ to calibrate the distribution of $W_{\text {critical }}^{p}(T)$ at room temperature and subsequently, fatigue life was predicted at eight different loading conditions, including multiple $\Delta \varepsilon$ and $R_{\varepsilon}$. In this work, we use all the fatigue life data that is available at a given temperature $T$ to obtain the distribution of
$W_{\text {critical }}^{p}(T)$ and repeat the process at multiple temperatures to characterize the temperature-dependent behavior of $W_{\text {critical }}^{p}(T)$.

A schematic of the Bayesian calibration approach for $W_{\text {critical }}^{p}(T)$ is shown in Fig. 7. Starting from the uniform prior distribution, the posterior distribution for $W_{\text {critical }}^{p}(T)$ at room temperature results in a normal distribution [33]. Therefore, in this work, we start with normal prior distributions of $W_{\text {critical }}^{p}(T)$ at a given temperature, $T$, and, subsequently, update the mean and the standard deviation of the distribution. With available $w_{N_{\mathrm{s}}}^{p}\left(\mathbf{x}^{*} ; \mathcal{B}\right)$ and $\Delta w_{N_{\mathrm{s}}}^{p}\left(\mathbf{x}^{*} ; \mathcal{B}\right)$ from the CPFE simulations in 20 SEMs and the prior distribution of $W_{\text {critical }}^{p}(T)$, we predict the distribution of fatigue life using Eq. (19). Subsequently, we compare the predicted and experimental fatigue life distributions. Based on this comparison, we update the distribution of $W_{\text {critical }}^{p}(T)$ using a combination of the Bayesian inference method, Metropolis-Hastings (MH) algorithm, and Markov chain Monte Carlo (MCMC) sampling. In the following paragraphs, we discuss the details for the comparison of the predicted and experimental fatigue life data. Subsequently, we describe the methodology to obtain the posterior distribution of $W_{\text {critical }}^{p}(T)$ using a combination of the Bayesian inference method, $\mathrm{MH}$ algorithm, and MCMC sampling.

\section{Comparison of the Experimental and Predicted Fatigue Life}

The experimental $\left(N_{\mathrm{f}}^{\text {expt }}(\mathcal{B})\right)$ and predicted $\left(N_{\mathrm{f}}^{\text {predict }}(\mathcal{B})\right)$ fatigue life can be related as

$N_{\mathrm{f}}^{\text {expt }}(\mathcal{B})=N_{\mathrm{f}}^{\text {predict }}\left(W_{\text {critical }}^{p}(T) ; \mathcal{B}\right)+\delta(\mathcal{B})+e(\mathcal{B})$

where $\delta(\mathcal{B})$ is the error associated with the model's inability to capture the correct physics, including limitations related to the phenomenological evolution equations of the crystal plasticity model; $e(\mathcal{B})$ is the error associated with the experimental measurements and uncertainty of fatigue life. Without any loss of generality, both error terms can be assumed to be independent and identically distributed (IID) random variables with zero mean and finite standard deviations. Hence, their summation is also an IID random variable with zero mean and a finite standard deviation, say $s(\mathcal{B})$. Clearly, by Eq. (21), a knowledge of the parameter $s(\mathcal{B})$ is necessary to decide if a distribution of $N_{\mathrm{f}}^{\text {predict }}\left(W_{\text {critical }}^{p}(T) ; \mathcal{B}\right)$ is acceptable for a given distribution of $N_{\mathrm{f}}^{\text {exp }}(\mathcal{B})$. However, $s(\mathcal{B})$ is also unknown in the beginning. Therefore, we need to seek an augmented parameter set $\mathcal{A}$, where $\mathcal{A}=\left\{W_{\text {critical }}^{p}(T), \mathrm{s}(\mathcal{B})\right\}$. We emphasize that the fatigue life model, given by Eq. (19), is still dependent on one parameter, namely $W_{\text {critical }}^{p}(T)$. Because of the present choice of the Bayesian approach for parameter calibration, we need to 

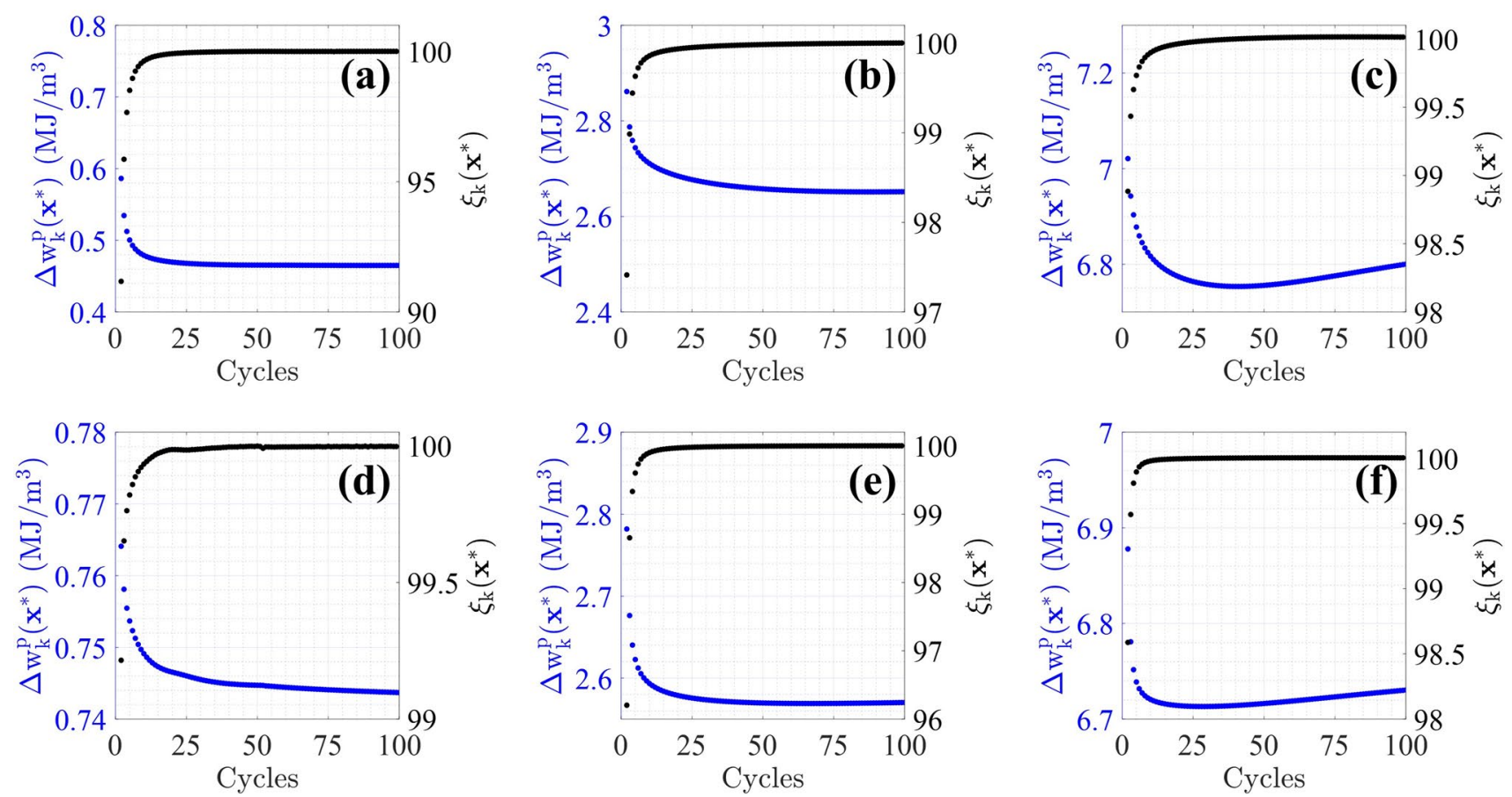

Fig. 8 Evolution of the plastic strain energy density per cycle with the number of cycles at the critical location within one of the SEMs under macroscopically applied strain, $\Delta \varepsilon=0.4 \%$ (left column), $0.6 \%$

(middle column), $0.75 \%$ (right column), and temperature, $T=20^{\circ} \mathrm{C}$ (top row), $750{ }^{\circ} \mathrm{C}$ (bottom row)

seek $W_{\text {critical }}^{p}(T)$ and $s(\mathcal{B})$ simultaneously. Once the posterior distribution of $W_{\text {critical }}^{p}(T)$ is known, the parameter $s(\mathcal{B})$ has no role to play in the fatigue life prediction.

\section{Updating Prior Distribution of $W_{\text {critical }}^{p}(T)$}

In the Bayesian inference method, Bayes' theorem is used to update the prior distribution of the parameter set $\mathcal{A}$ based on the experimental evidence and likelihood function to result in the posterior distribution as

$\pi(\mathcal{A} \mid \mathcal{D}(\mathcal{B}))=\frac{\pi(\mathcal{D}(\mathcal{B}) \mid \mathcal{A}) \pi_{0}(\mathcal{A})}{\pi(\mathcal{D}(\mathcal{B}))}=\frac{\pi(\mathcal{D}(\mathcal{B}) \mid \mathcal{A}) \pi_{0}(\mathcal{A})}{\int \pi(\mathcal{D}(\mathcal{B}) \mid \mathcal{A}) \pi_{0}(\mathcal{A}) \mathrm{d} \mathcal{A}}$

In Eq. (22), $\pi_{0}(\mathcal{A})$ is the prior distribution of the parameter set $\mathcal{A} ; \pi(\mathcal{D}(\mathcal{B}) \mid \mathcal{A})$ is the likelihood of observing experimental data, $\mathcal{D}(\mathcal{B})$, for the given parameter set $\mathcal{A} ; \pi(\mathcal{A} \mid \mathcal{D}(\mathcal{B}))$ is the posterior distribution of the parameter set $\mathcal{A}$ given the experimental evidence $\mathcal{D}(\mathcal{B})$ and likelihood term $\pi(\mathcal{D}(\mathcal{B}) \mid \mathcal{A})$. Since $\delta(\mathcal{B})$ and $e(\mathcal{B})$ in Eq. (21), are assumed to be IID random variables, the likelihood term in Eq. (22) can be expressed as [83]
$\pi(\mathcal{D}(\mathcal{B}) \mid \mathcal{A})=\frac{1}{\left(2 \pi s(\mathcal{B})^{2}\right)^{\frac{q}{2}}} \exp \left(-\frac{\operatorname{SSE}(\mathcal{A})}{2 s(\mathcal{B})^{2}}\right)$

where $s(\mathcal{B})$ is defined before; $\operatorname{SSE}(\mathcal{A})$ is the sum of squares of errors for the parameter set $\mathcal{A}$ and expressed as

$\operatorname{SSE}(\mathcal{A})=\sum_{i=1}^{n} \sum_{j=1}^{k}\left(N_{i}^{\text {expt }}(\mathcal{B})-N_{j}^{\text {predict }}(\mathcal{A} ; \mathcal{B})\right)^{2}$

In Eq. (24), $N_{i}^{\text {expt }}(\mathcal{B})$ is the $i$ th experimental fatigue life in a set of $n$ data, and $N_{j}^{\text {predict }}(\mathcal{A} ; \mathcal{B})$ is the predicted life corresponding to the $j$ th SEM in a set of $k$ (=20 in this work) SEMs. The parameter $q$ in Eq. (23) is related to $n$ and $k$ as

$q=n \cdot k$

Typically, it is challenging to evaluate the integral in the denominator of Eq. (22) analytically or using numerical quadrature. The MCMC sampling method is preferred in such situations to obtain $\pi(\mathcal{A} \mid \mathcal{D}(\mathcal{B}))$. A Markov chain is a sequence of random variables satisfying the property that the $h$ th term in the sequence depends only on the $(h-1) t h$ term. Based on a proposal distribution, the $h$ th term is identified via Monte Carlo sampling and the decision whether the new proposed candidate point will be accepted as the $h$ th term in the sequence is taken based on the MH algorithm $[84,85]$. 


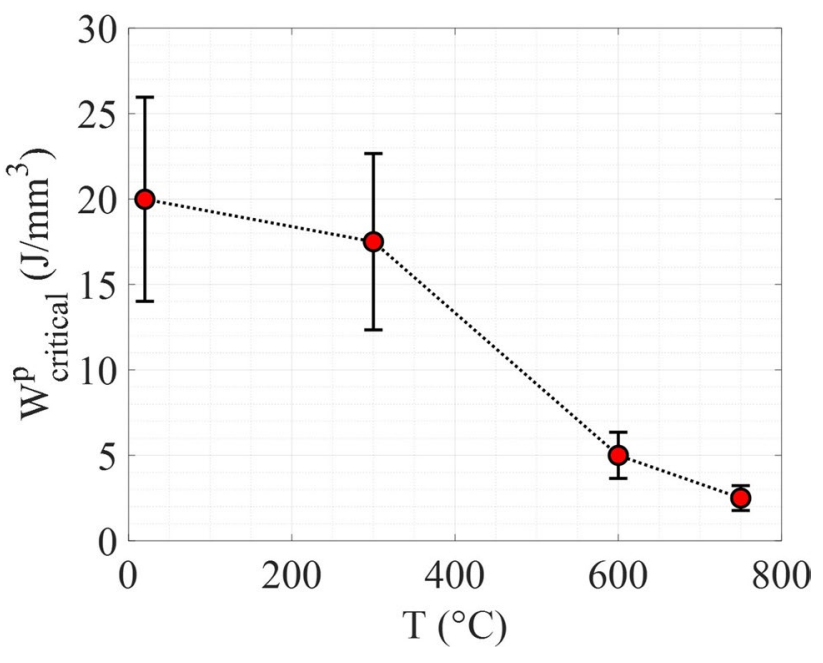

Fig. 9 Variation of $W_{\text {critical }}^{p}(T)$ as a function of temperature

Let, $\mathcal{A}_{r}$ be the $r$ th candidate in $\mathcal{A}$, where $\mathcal{A}=\left\{W_{\text {critical }}^{p}, \mathrm{~s}(\mathcal{B})\right\} ; \mathcal{A}_{r}^{h-1}$ be the same parameter in the $(h-1)$ th term of the Markov chain of $\mathcal{A} ; \mathcal{A}_{r}^{*}$ be the proposed $r$ th parameter for the $h t h$ term in the sequence. Now, we compute the following number:

$\mathcal{R}=\min \left(1, \frac{\pi\left(\mathcal{D}(\mathcal{B}) \mid \mathcal{A}_{r}^{*}\right) \pi_{0}\left(\mathcal{A}_{r}^{*}\right) \pi\left(\mathcal{A}_{r}^{*} \mid \mathcal{A}_{r}^{h-1}\right)}{\pi\left(\mathcal{D}(\mathcal{B}) \mid \mathcal{A}_{r}^{h-1}\right) \pi_{0}\left(\mathcal{A}_{r}^{h-1}\right) \pi\left(\mathcal{A}_{r}^{h-1} \mid \mathcal{A}_{r}^{*}\right)}\right)$

where $\pi\left(\mathcal{A}_{r}^{*} \mid \mathcal{A}_{r}^{h-1}\right)$ is the proposal distribution used to generate $\mathcal{A}_{r}^{*}$ based on $\mathcal{A}_{r}^{h-1}$. If the proposal distribution is symmetric $^{2^{r}}$

$\pi\left(\mathcal{A}_{r}^{*} \mid \mathcal{A}_{r}^{h-1}\right)=\pi\left(\mathcal{A}_{r}^{h-1} \mid \mathcal{A}_{r}^{*}\right)$

and consequently, Eq. (26) reduces to

$p=\min \left(1, \frac{\pi\left(\mathcal{D}(\mathcal{B}) \mid \mathcal{A}_{r}^{*}\right) \pi_{0}\left(\mathcal{A}_{r}^{*}\right)}{\pi\left(\mathcal{D}(\mathcal{B}) \mid \mathcal{A}_{r}^{h-1}\right) \pi_{0}\left(\mathcal{A}_{r}^{h-1}\right)}\right)$

Now, the MH algorithm is invoked to decide if $\mathcal{A}_{r}^{*}$ can be accepted as the $h$ th candidate in the sequence. Particularly, a uniformly distributed random number $u$ is generated within the interval $0 \leq u \leq 1$. If $u \leq p, \mathcal{A}_{r}^{h}=\mathcal{A}_{r}^{*}$; otherwise, $\mathcal{A}_{r}^{h}=\mathcal{A}_{r}^{h-1}$. This process is continued until convergence is achieved.

$\mathcal{M}$ Markov chains are run in parallel to check convergence. Let, $B_{\mathcal{A}_{r}}$ and $W_{\mathcal{A}_{r}}$ be the variance of the parameter $\mathcal{A}_{r}$ between and within chains, respectively. An estimate

\footnotetext{
${ }^{2}$ If the posterior distribution is expected to be symmetric, as in the present work, the assumption of symmetric proposal distribution leads to quick convergence.
}

of the variance of the parameter $\mathcal{A}_{r}, V_{\mathcal{A}_{r}}$, can be computed as

$V_{\mathcal{A}_{r}}=\frac{\mathcal{M}-1}{\mathcal{M}} W_{\mathcal{A}_{r}}+\frac{1}{\mathcal{M}} B_{\mathcal{A}_{r}}$

Now, the convergence parameter $R_{\mathcal{A}_{r}}$ is defined as

$R_{\mathcal{A}_{r}}=\sqrt{\frac{V_{\mathcal{A}_{r}}}{W_{\mathcal{A}_{r}}}}$

With the increase in Monte Carlo sampling, $h$, in each Markov chain, the parameter $R_{\mathcal{A}_{r}}$ approaches unity resulting in a converged/stationary distribution, also known as the posterior distribution. The readers may refer to Cross et al. [86] for the expressions of $W_{\mathcal{A}_{r}}$ and $B_{\mathcal{A}_{r}}$.

\section{Results}

To predict fatigue life and associated failure mode as a function of $\Delta \varepsilon$ and $T$, we consider the $R_{\varepsilon}=0$ regime and $T=20^{\circ} \mathrm{C}, 300^{\circ} \mathrm{C}, 600^{\circ} \mathrm{C}$, and $750^{\circ} \mathrm{C}$ temperatures. For each temperature, CPFE simulations are carried out at $\Delta \varepsilon=0.4 \%, 0.6 \%$, and $0.75 \%$. Subsequently, $w_{N_{\mathrm{s}}}^{p}\left(\mathbf{x}^{*} ; \mathcal{B}\right)$ and $\Delta w_{N_{\mathrm{s}}}^{p}\left(\mathbf{x}^{*} ; \mathcal{B}\right)$, as defined in section "Fatigue Life Prediction Model”, are nonlocally averaged as per Eq. (15). Using nonlocally averaged $w_{N_{\mathrm{s}}}^{p}\left(\mathbf{x}^{*} ; \mathcal{B}\right)$ and $\Delta w_{N_{\mathrm{s}}}^{p}\left(\mathbf{x}^{*} ; \mathcal{B}\right)$, and experimental fatigue life data for RR1000 for each simulated loading conditions, $W_{\text {critical }}^{p}(T)$ is calibrated for RR1000 as a function of temperature using the Bayesian calibration framework described in section "Bayesian Calibration of the Critical Plastic Strain Energy Density". Finally, using the calibrated $W_{\text {critical }}^{p}(T)$ values, and $w_{N_{\mathrm{s}}}^{p}\left(\mathbf{x}^{*} ; \mathcal{B}\right)$ and $\Delta w_{N_{\mathrm{s}}}^{p}\left(\mathbf{x}^{*} ; \mathcal{B}\right)$ from the CPFE simulations, fatigue life and associated failure modes are predicted. With this, the calibrated results for $W_{\text {critical }}^{p}(T)$ and comparison of the model prediction with experimental evidence are reported below.

\section{Critical Accumulated Plastic Strain Energy Density as a Function of Temperature}

As representative examples, the evolution of $\Delta w_{k}^{p}\left(\mathbf{x}^{*} ; \mathcal{B}\right)$ with the number of cycles, $k$, at the critical location $\left(\mathbf{x}^{*}\right)$ within one of the 20 SEMs, is shown in Fig. 8. ${ }^{3}$ It is evident from Fig. 8 that the saturation of $\Delta w_{k}^{p}\left(\mathbf{x}^{*} ; \mathcal{B}\right)$ is inherently dependent on $\Delta \varepsilon$ and $T$, i.e., $\mathcal{B}$. For the current set of 20 SEMs, each containing $\sim 2.5 \mathrm{M}$ elements, it is impractical to carry

\footnotetext{
3 Typically, $\Delta w_{1}^{p}\left(\mathbf{x}^{*} ; \mathcal{B}\right) \gg \Delta w_{k}^{p}\left(\mathbf{x}^{*} ; \mathcal{B}\right) \forall k>1$. Therefore, in Fig. 8, data points are shown starting from the second cycle to better visualize the cyclic evolution of $\Delta w_{k}^{p}\left(\mathbf{x}^{*} ; \mathcal{B}\right)$.
} 
out CPFE simulations for hundreds of cycles. Under such situations, researchers either continue simulations up to a point, usually three to 20 cycles, where the saturation of the fatigue indicative metric is acceptable $[9,11,13,14,22,23$, $26,31-34]$ or adopt accelerated simulation procedures based on the method of multiple time-scales [87-90] or perform reduced-order CPFE simulations [91]. The first approach has been widely used in the literature and is considered in this work. In such an approach, we define the degree of saturation at the $k$ th cycle, $\xi_{k}\left(\mathbf{x}^{*} ; \mathcal{B}\right)$, as

$\xi_{k}\left(\mathbf{x}^{*} ; \mathcal{B}\right)=\frac{\Delta w_{k+1}^{p}\left(\mathbf{x}^{*} ; \mathcal{B}\right)}{\Delta w_{k}^{p}\left(\mathbf{x}^{*} ; \mathcal{B}\right)} \times 100 \%$

The degree of saturation is considered to be acceptable if $\xi_{k}\left(\mathbf{x}^{*} ; \mathcal{B}\right) \geq 99.9 \%$. With this, for $\Delta \varepsilon=0.6 \%$ and $0.75 \%$, the simulations are continued up to 10 cycles; for $\Delta \varepsilon=0.4 \%$, simulations are extended up to 20 cycles. Consequently, $N_{\mathrm{s}}=10$ for $\Delta \varepsilon=0.6 \%$ and $0.75 \% ; N_{\mathrm{s}}=20$ for $\Delta \varepsilon=0.4 \%$.

For the Bayesian calibration of $W_{\text {critical }}^{p}(T)$, the CPFE simulations are performed on all SEMs at all $\Delta \varepsilon$ and $T$, as prescribed above. Subsequently, nonlocally averaged $w_{N_{\mathrm{s}}}^{p}\left(\mathbf{x}^{*} ; \mathcal{B}\right)$ and $\Delta w_{N_{\mathrm{s}}}^{p}\left(\mathbf{x}^{*} ; \mathcal{B}\right)$ are obtained. As described in section "Bayesian Calibration of the Critical Plastic Strain Energy Density", normal distributions are assumed as prior distributions for $W_{\text {critical }}^{p}(T)$ for all $T$. Subsequently, for each $T$, MCMC simulations are continued until $10^{5}$ iterations to ensure convergence. The mean value of $W_{\text {critical }}^{p}(T)$, obtained from the posterior distribution, is plotted in Fig. 9 as a function of temperature.

The length of the error bar in Fig. 9, say $\eta(\mathcal{B})$, represents the variability in $W_{\text {critical }}^{p}(T)$ and is computed as

$\eta(\mathcal{B})=\eta_{1}(\mathcal{B})+\eta_{2}(\mathcal{B})$

where $\eta_{1}(\mathcal{B})$ is the standard deviation of $W_{\text {critical }}^{p}(T)$ associated with the posterior distribution from the Bayesian calibration and $\eta_{2}(\mathcal{B})$ is the additional variability in $W_{\text {critical }}^{p}(T)$ due to the imprecise calibration of the CP parameters. Let $f(\zeta ; T)$ be the posterior probability density of $W_{\text {critical }}^{p}(T)=\zeta$ at the temperature $T$. Hence,

$\eta_{1}(\mathcal{B})=\sqrt{\int_{-\infty}^{\infty}\left[\zeta-\operatorname{mean}\left(W_{\text {critical }}^{p}(T)\right)\right]^{2} f(\zeta ; T) \mathrm{d} \zeta}$

where mean $\left(W_{\text {critical }}^{p}(T)\right)$ is the mean value of $W_{\text {critical }}^{p}(T)$ and is given by

$\operatorname{mean}\left(W_{\text {critical }}^{p}(T)\right)=\int_{-\infty}^{\infty} \zeta f(\zeta ; T) \mathrm{d} \zeta$
Ideally, one would expect $\eta_{1}(\mathcal{B})$ to automatically include $\eta_{2}(\mathcal{B})$. However, $w_{N_{\mathrm{s}}}^{p}\left(\mathbf{x}^{*} ; \mathcal{B}\right)$ and $\Delta w_{N_{\mathrm{s}}}^{p}\left(\mathbf{x}^{*} ; \mathcal{B}\right)$ are computed for a given set of CP parameters and are used as input via Eqs. (19) and (21) in the Bayesian calibration of $W_{\text {critical }}^{p}(T)$. Hence, $\eta_{1}(\mathcal{B})$ does not account for the variability associated with the CP parameters, and we need to consider $\eta_{2}(\mathcal{B})$ separately.

The computation of $\eta_{2}(\mathcal{B})$ is nontrivial. Bandyopadhyay et al. [57] have shown that the coefficient of variation of APSED at the critical location within an SEM due to the variability associated with the CP parameters is $\sim 0.25$. Their analysis was performed at room temperature and for $\Delta \varepsilon=1.0 \%$. Also, the CP constitutive equations, especially the reference stress evolution, g, is not identical to Ref. [57]. Hence, the result from Ref. [57] may not be precisely applicable in the present context, but at least indicative of the possible uncertainty in $w_{N_{\mathrm{f}}}^{p}\left(\mathbf{x}^{*} ; \mathcal{B}\right)$ for the current CPFE simulations of RR1000. From this assessment, due to the uncertainty in the CP parameter, the coefficient of variation of $w_{N_{\mathrm{f}}}^{p}\left(\mathbf{x}^{*} ; \mathcal{B}\right)$ and thereby the calibrated $W_{\text {critical }}^{p}$ would be approximately 0.25 . Therefore,

$\eta_{2}(\mathcal{B}) \approx 0.25$ mean $\left(W_{\text {critical }}^{p}(T)\right)$

In Fig. 9, the uncertainty in $W_{\text {critical }}^{p}(T)$, i.e., $\eta(\mathcal{B})$, appears to decrease with increasing temperature, which is because $\eta_{2}(\mathcal{B})$ is proportional to the mean $\left(W_{\text {critical }}^{p}(T)\right)$ (see Eq. 35). Hence, as the mean $\left(W_{\text {critical }}^{p}(T)\right)$ decreases, $\eta_{2}(\mathcal{B})$ also decreases monotonically. As mentioned before, we have made a simplifying assumption that the proportionality constant in Eq. (35) is independent of $\mathcal{B}$. For a more rigorous estimation of the uncertainty in $W_{\text {critical }}^{p}(T)$, one needs to repeat the analysis in Ref. [57] at multiple loading conditions to obtain the proportionality constant as a function of $\mathcal{B}$. Such a task is left as future work.

The monotonically decreasing trend of $W_{\text {critical }}^{p}(T)$ with increasing temperature (see Fig. 9) is consistent with the fatigue strength of a material decreasing at elevated temperatures. Pan et al. also confirm such a decreasing trend with their critical stored energy criteria for crack nucleation [92]. Additionally, in Fig. 9, we note that $W_{\text {critical }}^{p}(T)$ appears to decrease along a vertically reflected sigmoidal curve with increasing temperature. However, further research and more fatigue data at additional temperature values, especially in the $300^{\circ} \mathrm{C}<T<600^{\circ} \mathrm{C}$ interval, are necessary to understand the exact shape and underlying physics behind such a trend and establish a temperature-dependent functional form for $W_{\text {critical }}^{p}(T)$. 

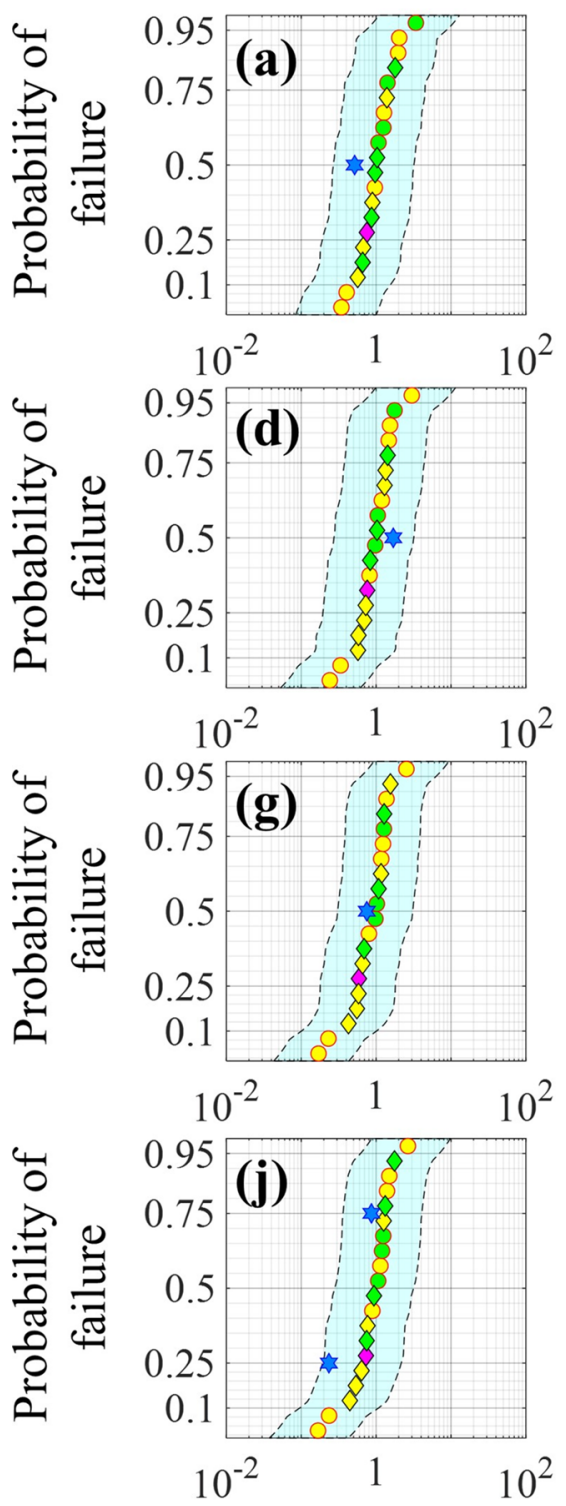

Normalized fatigue life

Microstructure without inclusion, MDF, free surface

Microstructure without inclusion, MDF, interior

Microstructure with inclusion, IDF, interior

Experimental data, MDF
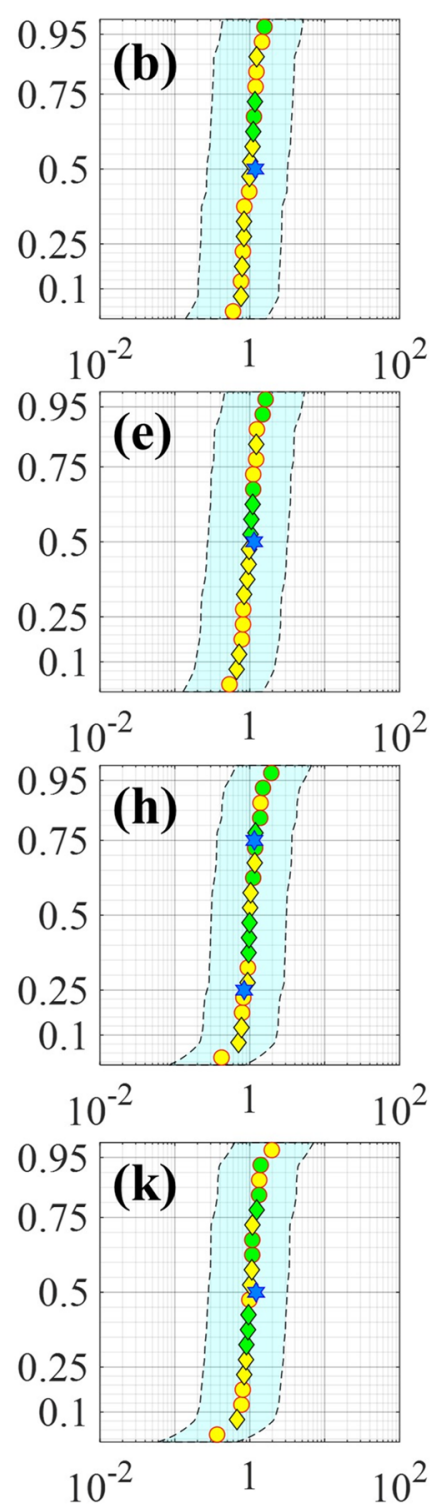

Normalized fatigue life

$\diamond \quad$ Microstructure with inclusion, $\mathrm{MDF}$, free surface

Microstructure with inclusion, MDF, interior

Prediction range based on 95\% confidence level of $\mathrm{W}_{\text {critical }}^{\mathrm{p}}, \Delta \mathrm{w}_{\mathrm{N}_{\mathrm{s}}}^{\mathrm{p}}\left(\mathbf{x}^{*} ; \mathcal{B}\right)$, and $\mathrm{w}_{\mathrm{N}_{\mathrm{s}}}^{\mathrm{p}}\left(\mathbf{x}^{*} ; \boldsymbol{B}\right)$ 
4Fig. 10 The probability of failure plots considering log-normal distribution as reference. In each subplot, fatigue life is normalized with respect to the median predicted life. Each row corresponds to a constant temperature with $T=20^{\circ} \mathrm{C}$ for the first row (from top), $T=300^{\circ} \mathrm{C}$ for the second row, $T=600^{\circ} \mathrm{C}$ for the third row, and $T=750^{\circ} \mathrm{C}$ for the fourth row. Each column corresponds to a constant applied strain range with $\Delta \varepsilon=0.4 \%$ for the first column (from left), $\Delta \varepsilon=0.6 \%$ for the second column, and $\Delta \varepsilon=0.75 \%$ for the third column. MDF stands for matrix-driven failure, and IDF stands for inclusion-driven failure. 'free surface' represents failure originating from the free surface of the microstructure, and 'interior' represents cases where failure originates within the bulk of the microstructure (a)

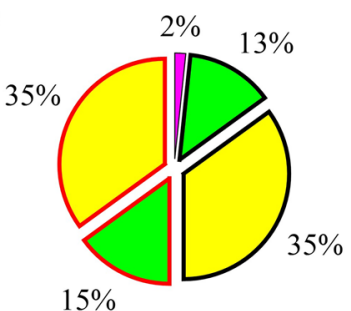

(c)

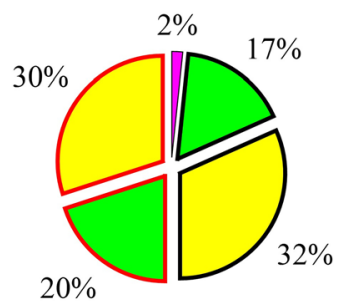

(b)

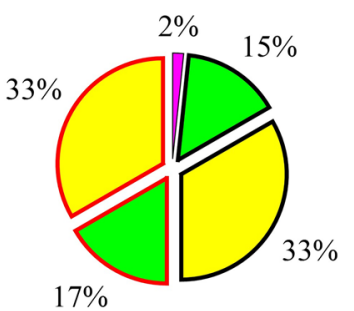

(d)

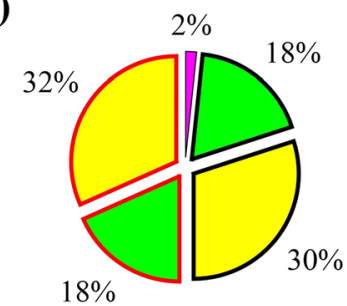

$\square$ SEM w/o inclusion, MDF (Free surface)

$\square$ SEM w/o inclusion, MDF (Interior)

$\square$ SEM w/ inclusion, MDF (Free surface)

$\square$ SEM w/ inclusion, MDF (Interior)

$\square$ SEM w/ inclusion, IDF (Subsurface inclusion)

Fig. 11 Pie charts showing the percentage of different predicted failure modes at $\mathbf{a} 20^{\circ} \mathrm{C}, \mathbf{b} 300^{\circ} \mathrm{C}, \mathbf{c} 600^{\circ} \mathrm{C}$, and $\mathbf{d ~} 750{ }^{\circ} \mathrm{C}$. MDF stands for matrix-driven failure, and IDF stands for inclusion-driven failure. 'Free surface' represents failure originating from the free surface of the microstructure, and 'Interior' represents cases where the failure arises within the bulk of the microstructure

\section{Prediction of Fatigue Life and Associated Failure Modes}

The simulated and nonlocally averaged $w_{N_{\mathrm{s}}}^{p}\left(\mathbf{x}^{*} ; \mathcal{B}\right)$ and $\Delta w_{N_{\mathrm{s}}}^{p}\left(\mathbf{x}^{*} ; \mathcal{B}\right)$ values from every SEM at each loading conditions are used within Eq. (19) along with the mean values of $W_{\text {critical }}^{p}(T)$, as shown in Fig. 9, to predict fatigue lives and the associated failure modes. Specifically, for SEMs with inclusions, if $\mathbf{x}^{*}$ is located within the distance, $d_{\mathrm{eq}}$, from the centroid of the inclusion, where $d_{\text {eq }}$ is the equivalent diameter of the inclusion, such a situation would indicate an IDF; otherwise, the simulation predicts an MDF. The probability of failure plots are obtained using the probplot function in MATLAB, considering a log-normal distribution as a reference and shown in Fig. 10. The normalized fatigue life is plotted in Fig. 10, where the normalized factor is the ratio of the actual life and median life, whereas a life of 10 on the scale represents a factor of 10 multiplied to the median life.

As previously shown [57], this framework, using the $W_{\text {critical }}^{p}(T)$, is capable of predicting log-normally distributed fatigue life at room temperature, and the predicted scatter increases with decreasing $\Delta \varepsilon$. Both characteristics of the predicted life distributions are emergent behavior from the model and indicate the physical nature of fatigue behavior. In this work, a similar observation is also made in Fig. 10. For example, (a) the predicted data points in each subplot in Fig. 10 appear to be aligned along a straight line, confirming that the predicted fatigue life follows a log-normal distribution and (b) for each temperature, scatter in the predicted fatigue life increases with decreasing $\Delta \varepsilon$.

In section "Critical Accumulated Plastic Strain Energy Density as a Function of Temperature", we quantified the variability in $W_{\text {critical }}^{p}(T)$, i.e., $\eta(\mathcal{B})$, using Eqs. (32)-(35). We also mentioned that the variability in the simulated $w_{N_{\mathrm{s}}}^{p}\left(\mathbf{x}^{*} ; \mathcal{B}\right)$ and $\Delta w_{N_{\mathrm{s}}}^{p}\left(\mathbf{x}^{*} ; \mathcal{B}\right)$ values within an SEM is unavoidable due to the uncertainty in the $\mathrm{CP}$ parameters. Following the discussion in section "Critical Accumulated Plastic Strain Energy Density as a Function of Temperature", the standard deviation in $w_{N_{\mathrm{s}}}^{p}\left(\mathbf{x}^{*} ; \mathcal{B}\right)$ and $\Delta w_{N_{\mathrm{s}}}^{p}\left(\mathbf{x}^{*} ; \mathcal{B}\right)$ values are approximately 0.25 times their mean values. Thus, the knowledge of the mean and the standard deviation of $W_{\text {critical }}^{p}(T), w_{N_{\mathrm{s}}}^{p}\left(\mathbf{x}^{*} ; \mathcal{B}\right)$, and $\Delta w_{N_{\mathrm{s}}}^{p}\left(\mathbf{x}^{*} ; \mathcal{B}\right)$ enables us to compute the lower and upper bounds of these quantities corresponding to their $95 \%$ confidence interval, ${ }^{4}$ which can subsequently be used in Eq. (19) to evaluate a prediction interval for the fatigue life. Specifically, one can obtain the lower bound of the prediction interval by plugging the lower bounds of $W_{\text {critical }}^{p}(T)$ and $w_{N_{\mathrm{s}}}^{p}\left(\mathbf{x}^{*} ; \mathcal{B}\right)$, and upper bound of $\Delta w_{N_{\mathrm{s}}}^{p}\left(\mathbf{x}^{*} ; \mathcal{B}\right)$ into Eq. (19). Specifically, the upper prediction bound is computed as

$\left.N_{\mathrm{f}}^{\text {predict }}\right|_{\text {ub }}=\frac{\left(W_{\text {critical }}^{p}(T)+2 \eta(\mathcal{B})\right)-(1-2 \times 0.25) \times w_{N_{s}}^{p}\left(\mathbf{x}^{*} ; \mathcal{B}\right)}{(1-2 \times 0.25) \times \Delta w_{N_{\mathrm{s}}}^{p}\left(\mathbf{x}^{*} ; \mathcal{B}\right)}+N_{\mathrm{s}}(\mathcal{B})$

and the lower prediction bound is computed as

$\left.N_{\mathrm{f}}^{\text {predict }}\right|_{\mathrm{lb}}=\frac{\left(W_{\text {critical }}^{p}(T)-2 \eta(\mathcal{B})\right)-(1+2 \times 0.25) \times w_{N_{\mathrm{s}}}^{p}\left(\mathbf{x}^{*} ; \mathcal{B}\right)}{(1+2 \times 0.25) \times \Delta w_{N_{\mathrm{s}}}^{p}\left(\mathbf{x}^{*} ; \mathcal{B}\right)}+N_{\mathrm{s}}(\mathcal{B})$

Such a fatigue life prediction interval is shown in Fig. 10 as a shaded region and is based on a $95 \%$ confidence level of $W_{\text {critical }}^{p}(T), w_{N_{\mathrm{s}}}^{p}\left(\mathbf{x}^{*} ; \mathcal{B}\right)$, and $\Delta w_{N_{\mathrm{s}}}^{p}\left(\mathbf{x}^{*} ; \mathcal{B}\right)$.

\footnotetext{
${ }^{4}$ If $\mu$ and $\sigma$ are the mean and the standard deviation of a normally distributed random variable, the $95 \%$ confidence interval can be approximated as $[\mu-2 \sigma, \mu+2 \sigma]$.
} 
Fig. 12 a Comparison of the percentage of free surface failure from simulation and experiments. Percentage of inclusionand matrix-driven failures from b simulation and $\mathbf{c}$ experiment. MDF stands for matrix-driven failure, and IDF stands for inclusion-driven failure
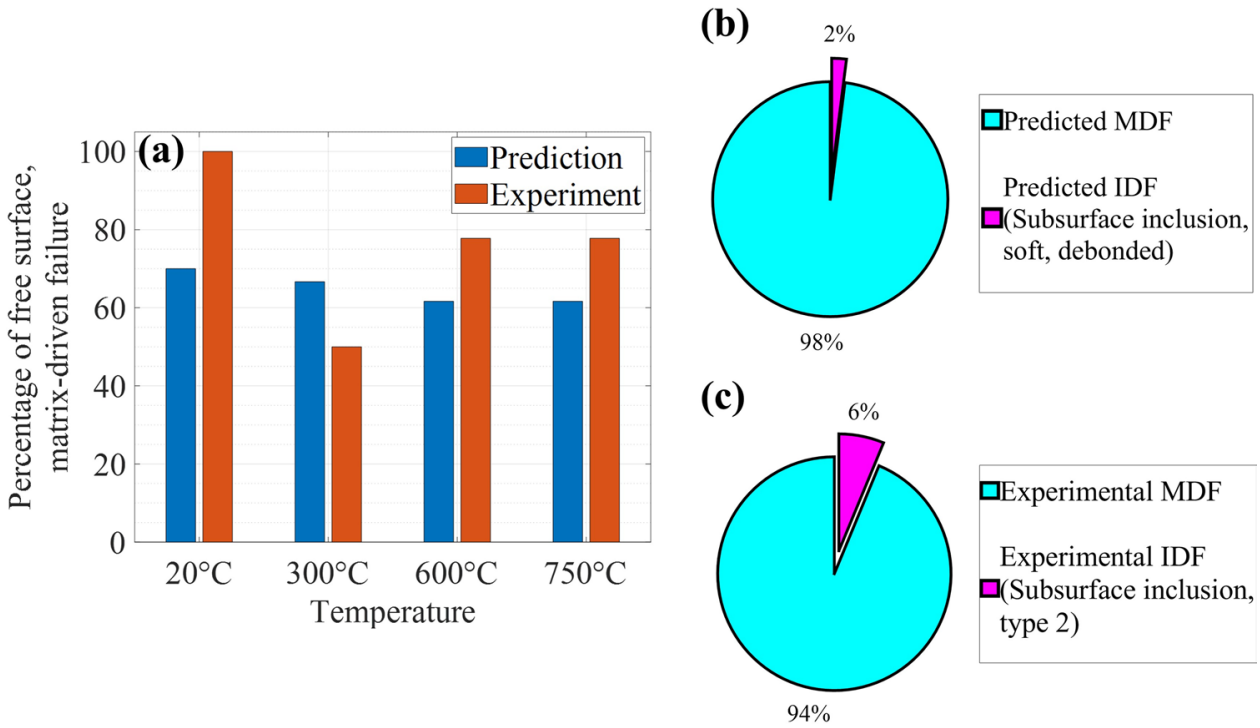

Although inclusions are present in 10 SEMs, only one IDF is predicted at $\Delta \varepsilon=0.4 \%$, consistently, at all temperatures (see Fig. 10). Further, the fatigue life associated with the IDF does not correspond to the minimum life predicted from the simulations. In the present experimental dataset, evidence of IDF is only observed for $\Delta \varepsilon \leq 0.4 \%$, and the life associated with IDF does not correspond to the minimum life. Although $\Delta \varepsilon<0.4 \%$ regime is not explored in this study, however, based on the results of the prior parametric study and accompanied discussion therein [52], a higher probability of IDF is expected in the $\Delta \varepsilon<0.4 \%$ regime.

As mentioned in section "Background", a turbine disk produced with a DMHT shows fine grains near the bore region and coarse grain microstructure near the rim region. In experimental research with a turbine disk made of LSHR, Gabb et al. [93] showed that the fine-grained microstructure leads to higher fatigue life than the coarse grain microstructure. In their research, Type 2 IDF is observed in the fine-grained region, whereas crystallographic surface and subsurface facets are responsible for failure in the coarse grain region. The crystallographic facet resulted in the minimum fatigue life observed in their samples. The experimentally observed minimum life for the coarse grain variant of RR1000 is associated with MDF as well. From Fig. 10, one can verify that the predicted minimum lives in all simulated loading conditions correspond to an MDF. Further, the MDF corresponding to the minimum life originated from a microstructure without inclusion, thereby confirming that the minimum life is not necessarily always influenced by the presence of inclusions.

Gabb et al. [93] reported that the location of the failure, associated with MDF, shifted more towards the interior of the samples with increasing temperature as opposed to originating from the free or near free-surface. Further, failure originating at the free surface was associated with lower life than failure arising from the interior. Additionally, Hyzak and Bernstein [36] observed a change in the crack initiation location from the surface to the interior of the AF-115 and AF2-1DA specimens with decreasing strain range. The present fatigue life prediction framework captures these trends in temperature- and strain range-dependent behavior of failure location. For example, it can be seen in Fig. 10 that (a) the minimum life is always associated with the MDF originating from the free surface, (b) failure arising at the interior of the microstructures are, in general, associated with relatively higher fatigue life, (c) failure originates at the interior of the SEMs more frequently with decreasing strain range. Further, from Fig. 11, it is observed that the percentage of free surface failures decreases with increasing temperatures and thereby indicating the location of the failure, associated with MDF mode, shifting more towards the interior of the SEMs. From Fig. 12a, we can verify that such a trend is in good qualitative agreement with the experimental observation in RR1000.

From the simulation results in Fig. 11, the percentage of IDF remains unchanged with changing temperature. Such an observation is perhaps because the same set of SEMs is simulated across all temperatures. Overall, the modeling framework predicts $2 \% \mathrm{IDFs}^{5}$ (Fig. 12b). These failures originate in an SEM having a structurally soft, partially debonded, and subsurface inclusion. On the other hand, the present

\footnotetext{
5 As described in section "Material Characterization", the size and sphericity distributions of inclusion are characterized using $\mu-\mathrm{CT}$ within a $433 \mu \mathrm{m} \times 300 \mu \mathrm{m} \times 1250 \mu \mathrm{m}$ rectangular cuboid volume of the material. Therefore, the present work does not account for the statistical extremes in geometric attributes of the inclusions. These extremities may limit fatigue life.
} 
experimental dataset show 6\% $\mathrm{IDFs}^{6}$ (Fig. 12c), where all such failures originate from subsurface Type 2 inclusions. Although all inclusions in this work are geometrically modeled as ellipsoids, i.e., Type 1, a structurally soft inclusion with a partially debonded region would implicitly capture some of the effects of a Type 2 inclusion in the material. Thus, the predictions regarding the inclusion type associated with IDF are in good agreement with the experimental observations. Further, a recognized underestimation of the percentage of IDFs from the present modeling framework is attributed to the presence of a structurally soft inclusion with a partially debonded region, e.g., the worst-case scenario, in only one SEM. From the results, as presented above, it is clear that the microstructure-sensitive energybased approach [33] is capable of capturing the median and scatter trend in fatigue life data, as well as the multiple failure modes and realistic shift in the location of failure with varying applied strain range and temperature.

\section{Conclusions}

In this work, a microstructure-sensitive energy-based approach is used to predict the fatigue life, associated failure mode, and uncertainty in the fatigue life prediction as a function of applied strain range and temperature. The fatigue life prediction model used in this work involves only one material constant: the critical accumulated plastic strain energy density (APSED). Using a Bayesian inference method, the critical APSED is calibrated for a powder metallurgy produced Ni-base superalloy, namely RR1000, as a function of temperature. Subsequently, the calibrated critical APSED is used to predict inclusion- and matrix-driven competing failure modes in RR1000 as a function of applied strain range $(\Delta \varepsilon=0.4 \%, 0.6 \%$, and $0.75 \%)$ and temperature $\left(T=20^{\circ} \mathrm{C}\right.$, $300^{\circ} \mathrm{C}, 600^{\circ} \mathrm{C}$, and $750^{\circ} \mathrm{C}$ ) for $R_{\varepsilon}=0$ loading regime. Specifically, for fatigue life prediction, 20 statistically equivalent microstructures, 10 of these containing inclusions and the remaining 10 without inclusions, are used to conduct simulations using crystal plasticity finite element (CPFE). The key findings are summarized below.

- The microstructure-sensitive critical APSED decreases along a vertically reflected sigmoidal curve with increasing temperature, which is consistent with the fatigue strength of the material decreasing at the elevated temperature.

\footnotetext{
${ }^{6} 6 \%$ inclusion-driven failures from experiments is based on a relatively small sample of $\sim 100$ data points. One may expect a significantly higher percentage of inclusion-driven failures with a larger sample size encompassing additional loading conditions.
}

- The predicted fatigue life from the statistically equivalent microstructures follows a log-normal distribution at each simulated loading condition, which is consistent with the traditional fatigue experimental results. Moreover, the predicted scatter in fatigue life also increases with decreasing strain range for a given temperature.

- For each simulated loading condition, the uncertainty in fatigue life is quantified as a prediction interval computed based on the 95\% confidence level of the critical APSED and the simulated APSED from CPFE. Experimental data for each loading condition is seen to fall within such a prediction interval.

- From CPFE simulations, inclusion-driven failure is observed only at $\Delta \varepsilon=0.4 \%$ across all temperatures. Experimentally, inclusion-driven failures are only observed for $\Delta \varepsilon \leq 0.4 \%$. Although $\Delta \varepsilon<0.4 \%$ regime is not explored explicitly in this study, it has been previously shown in a parametric study [52] that there exists a higher probability of inclusion-driven failure in the $\Delta \varepsilon<0.4 \%$ regime.

- From the simulations, the inclusion-driven failure mode is not (necessarily) associated with the minimum life, which is consistent with experimental observation. Albeit, this observation may change with microstructure inputs that include larger inclusions (far greater than the average grain size of the material). Experimentally, the minimum life corresponds to matrix-driven failure. In simulations, minimum life also corresponds to matrixdriven failure and originates from a microstructure that does not contain an inclusion.

- In the literature, it is seen that the location of the failure, associated with matrix-driven failure, shifts towards the interior of the specimen from the free surface with increasing temperature, and the failures originating from the interior are typically associated with higher life. In simulations, the percentage of failure originating from the free surface is seen to be decreasing with increasing temperature as well. Further, life, associated with failure from the interior, is predicted to be consistently higher than the free surface failure life.

The above findings extend the framework proposed by Bandyopadhyay et al. [33] across various temperature regimes and competing failure modes while offering uncertainty quantification and propagation. Thus, this work provides a valuable step towards the adoption of microstructural-based modeling assessments for fatigue lifing.

Acknowledgements We sincerely appreciate the financial support received from Rolls-Royce Corporation to carry out the present research. We want to thank Mr. David E. Mills from Rolls-Royce Corporation; Dr. Mark Hardy, Dr. Christos Argyrakis, Dr. Duncan Maclachlan, and Dr. Han Tai from Rolls-Royce plc for useful discussions. 
We also thank Dr. Prithivirajan Veerappan from Purdue University for sharing the scripts to quantify the quality of C3D4 elements.

\section{Compliance with Ethical Standards}

Conflict of interest The authors declare that they have no conflict of interest.

Open Access This article is licensed under a Creative Commons Attribution 4.0 International License, which permits use, sharing, adaptation, distribution and reproduction in any medium or format, as long as you give appropriate credit to the original author(s) and the source, provide a link to the Creative Commons licence, and indicate if changes were made. The images or other third party material in this article are included in the article's Creative Commons licence, unless indicated otherwise in a credit line to the material. If material is not included in the article's Creative Commons licence and your intended use is not permitted by statutory regulation or exceeds the permitted use, you will need to obtain permission directly from the copyright holder. To view a copy of this licence, visit http://creativecommons.org/licenses/by/4.0/.

\section{References}

1. Reed RC (2006) The superalloys: fundamentals and applications

2. Detrois M, Rotella J, Hardy M, Tin S, Sangid MD (2017) Tailoring the properties of a Ni-based superalloy via modification of the forging process: an ICME approach to fatigue performance. Integr Mater Manuf Innov 6:265-278. https://doi.org/10.1007/ s40192-017-0103-6

3. Hardy MC, Detrois M, McDevitt ET, Argyrakis C, Saraf V, Jablonski PD, Hawk JA, Buckingham RC, Kitaguchi HS, Tin S (2020) Solving recent challenges for wrought Ni-base superalloys. Metall Mater Trans A 51:2626-2650. https://doi.org/10.1007/ s11661-020-05773-6

4. Mathey GF (1994) Method of making superalloy turbine disks having graded coarse and fine grains. U.S. Patent No. 5,312,497, 17 May 1994

5. Gayda J, Furrer D (2003) Dual-microstructure heat treatment. Adv Mater Process 36-39

6. Bandyopadhyay R (2020) Ensuring fatigue performance via location-specific lifing in aerospace components made of titanium alloys and nickel-base superalloys. Purdue University Graduate School

7. McDowell DL (2005) Microstructure-sensitive computational fatigue analysis. In: Yip S (ed) Handbook of materials modeling. Springer, Berlin, pp 1193-1214

8. Shenoy MM, Gordon AP, McDowell DL, Neu RW (2005) Thermomechanical fatigue behavior of a directionally solidified Nibase superalloy. J Eng Mater Technol 127:325-336. https://doi. org/10.1115/1.1924560

9. Shenoy M, Zhang J, McDowell DL (2007) Estimating fatigue sensitivity to polycrystalline Ni-base superalloy microstructures using a computational approach. Fatigue Fract Eng Mater Struct 30:889-904. https://doi.org/10.1111/j.1460-2695.2007.01159.x

10. Xue Y, McDowell DL, Horstemeyer MF, Dale MH, Jordon JB (2007) Microstructure-based multistage fatigue modeling of aluminum alloy 7075-T651. Eng Fract Mech 74:2810-2823. https ://doi.org/10.1016/j.engfracmech.2006.12.031

11. Przybyla CP, Prasannavenkatesan R, Salajegheh N, McDowell DL (2010) Microstructure-sensitive modeling of high cycle fatigue. Int J Fatigue 32:512-525. https://doi.org/10.1016/j.ijfat igue.2009.03.021

12. Przybyla CP, McDowell DL (2010) Microstructure-sensitive extreme value probabilities for high cycle fatigue of Ni-base superalloy IN100. Int J Plast 26:372-394. https://doi.org/10.1016/j.ijpla s.2009.08.001

13. Przybyla CP, McDowell DL (2012) Microstructure-sensitive extreme-value probabilities of high-cycle fatigue for surface vs. subsurface crack formation in duplex Ti-6Al-4V. Acta Mater 60:293-305. https://doi.org/10.1016/j.actamat.2011.09.031

14. Przybyla CP, Musinski WD, Castelluccio GM, McDowell DL (2013) Microstructure-sensitive HCF and VHCF simulations. Int J Fatigue 57:9-27. https://doi.org/10.1016/j.ijfatigue.2012.09.014

15. Sinha S, Ghosh S (2006) Modeling cyclic ratcheting based fatigue life of HSLA steels using crystal plasticity FEM simulations and experiments. Int J Fatigue 28:1690-1704. https://doi. org/10.1016/j.ijfatigue.2006.01.008

16. Kirane K, Ghosh S (2008) A cold dwell fatigue crack nucleation criterion for polycrystalline Ti-6242 using grain-level crystal plasticity FE Model. Int J Fatigue 30:2127-2139. https://doi. org/10.1016/j.ijfatigue.2008.05.026

17. Anahid M, Samal MK, Ghosh S (2011) Dwell fatigue crack nucleation model based on crystal plasticity finite element simulations of polycrystalline titanium alloys. J Mech Phys Solids 59:21572176. https://doi.org/10.1016/j.jmps.2011.05.003

18. Sangid MD, Maier HJ, Sehitoglu H (2011) A physically based fatigue model for prediction of crack initiation from persistent slip bands in polycrystals. Acta Mater 59:328-341. https://doi. org/10.1016/j.actamat.2010.09.036

19. Sangid MD, Maier HJ, Sehitoglu H (2011) An energy-based microstructure model to account for fatigue scatter in polycrystals. J Mech Phys Solids 59:595-609. https://doi.org/10.1016/j. jmps.2010.12.014

20. Yeratapally SR, Glavicic MG, Hardy M, Sangid MD (2016) Microstructure based fatigue life prediction framework for polycrystalline nickel-base superalloys with emphasis on the role played by twin boundaries in crack initiation. Acta Mater 107:152-167. https://doi.org/10.1016/j.actamat.2016.01.038

21. Korsunsky AM, Dini D, Dunne FPE, Walsh MJ (2007) Comparative assessment of dissipated energy and other fatigue criteria. Int J Fatigue 29:1990-1995. https://doi.org/10.1016/j.ijfat igue.2007.01.007

22. Wan VVC, Maclachlan DW, Dunne FPE (2014) A stored energy criterion for fatigue crack nucleation in polycrystals. Int J Fatigue 68:90-102. https://doi.org/10.1016/j.ijfatigue.2014.06.001

23. Chen B, Jansenss K, Dunne FPE (2020) Role of geometrically necessary dislocation density in multiaxial and non-proportional fatigue crack nucleation. Int J Fatigue 135:105517. https://doi. org/10.1016/j.ijfatigue.2020.105517

24. Jiang J, Yang J, Zhang T, Zou J, Wang Y, Dunne FPE, Britton TB (2016) Microstructurally sensitive crack nucleation around inclusions in powder metallurgy nickel-based superalloys. Acta Mater 117:333-344. https://doi.org/10.1016/j.actamat.2016.07.023

25. Chen B, Jiang J, Dunne FPEE (2018) Is stored energy density the primary meso-scale mechanistic driver for fatigue crack nucleation? Int J Plast 101:213-229. https://doi.org/10.1016/j.ijpla s.2017.11.005

26. Bergsmo A, Dunne FPE (2020) Competing mechanisms of particle fracture, decohesion and slip-driven fatigue crack nucleation in a PM nickel superalloy. Int J Fatigue 135:105573. https://doi. org/10.1016/j.ijfatigue.2020.105573

27. Wilson D, Zheng Z, Dunne FPE (2018) A microstructure-sensitive driving force for crack growth. J Mech Phys Solids 121:147-174. https://doi.org/10.1016/j.jmps.2018.07.005

28. Wilson D, Dunne FPE (2019) A mechanistic modelling methodology for microstructure-sensitive fatigue crack growth. 
J Mech Phys Solids 124:827-848. https://doi.org/10.1016/j. jmps.2018.11.023

29. Wilson D, Wan W, Dunne FPE (2019) Microstructurally-sensitive fatigue crack growth in $\mathrm{HCP}, \mathrm{BCC}$ and FCC polycrystals. J Mech Phys Solids 126:204-225. https://doi.org/10.1016/j. jmps.2019.02.012

30. Lu X, Dunne FPE, Xu Y (2020) A crystal plasticity investigation of slip system interaction, GND density and stored energy in non-proportional fatigue in nickel-based superalloy. Int J Fatigue 139:105782. https://doi.org/10.1016/j.ijfatigue.2020.105782

31. Cruzado A, Lucarini S, LLorca J, Segurado J (2018) Microstructure-based fatigue life model of metallic alloys with bilinear Coffin-Manson behavior. Int J Fatigue 107(40): 48. https://doi. org/10.1016/j.ijfatigue.2017.10.014

32. Cruzado A, Lucarini S, LLorca J, Segurado J (2018) Crystal plasticity simulation of the effect of grain size on the fatigue behavior of polycrystalline Inconel 718. Int J Fatigue 113:236-245. https ://doi.org/10.1016/j.ijfatigue.2018.04.018

33. Bandyopadhyay R, Prithivirajan V, Peralta AD, Sangid MD (2020) Microstructure sensitive critical plastic strain energy density criterion for fatigue life prediction across various loading regimes. Proc R Soc A 476:20190766. https://doi.org/10.1098/ rspa.2019.0766

34. Prithivirajan V, Sangid MD (2020) Examining metrics for fatigue life predictions of additively manufactured IN718 via crystal plasticity modeling including the role of simulation volume and microstructural constraints. Mater Sci Eng A. https://doi. org/10.1016/j.msea.2020.139312

35. Morrow JD (1965) Cyclic plastic strain energy and fatigue of metals. Internal friction, damping, and cyclic plasticity, ASTM STP 378. American Society for Testing and Materials, Philadelphia, pp 45-87

36. Hyzak JM, Bernstein IM (1982) The effect of defects on the fatigue crack initiation process in two $\mathrm{p} / \mathrm{m}$ superalloys: part $\mathrm{i}$. fatigue origins. Metall Trans A 13:33-43. https://doi.org/10.1007/ BF02642413

37. Huron ES, Roth PG (1996) The influence of inclusions on low cycle fatigue life in a P/M nickel-base disk superalloy. Superalloys. https://doi.org/10.7449/1996/Superalloys_1996_359_368

38. Bhowal PR, Wusatowska-Sarnek AM (2005) Carbides and their influence on notched low cycle fatigue behavior of fine-grained IN718 gas turbine. In: Loria EA (ed) Superalloys 718, 625, 706 and derivatives. The Minerals, Metals and Materials Society, Pittsburgh, pp 341-349

39. Caton M, Jha S, Rosenberger A, Larsen J (2004) Divergence of mechanisms and the effect on the fatigue life variability of Rene' 88 DT. Superalloys. https://doi.org/10.7449/2004/Superalloy s_2004_305_312

40. Qiu C, Wu X (2014) High cycle fatigue and fracture behaviour of a hot isostatically pressed nickel-based superalloy. Philos Mag 94:242-264. https://doi.org/10.1080/14786435.2013.852287

41. Texier D, Cormier J, Villechaise P, Stinville JC, Torbet CJ, Pierret S, Pollock TM (2016) Crack initiation sensitivity of wrought direct aged alloy 718 in the very high cycle fatigue regime: the role of non-metallic inclusions. Mater Sci Eng A 678:122-136

42. Texier D, Gómez AC, Pierret S, Franchet JM, Pollock TM, Villechaise P, Cormier J (2016) Microstructural features controlling the variability in low-cycle fatigue properties of alloy Inconel 718DA at intermediate temperature. Metall Mater Trans A 47:1096-1109. https://doi.org/10.1007/s11661-015-3291-8

43. Shi Y, Yang D, Yang X, Miao G, Shi D, Li S, Hu X, Liu F, Huang W (2020) The effect of inclusion factors on fatigue life and fracture-mechanics-based life method for a P/M superalloy at elevated temperature. Int J Fatigue 131:105365. https://doi.org/10.1016/j. ijfatigue.2019.105365
44. Alexandre F, Deyber S, Pineau A (2004) Modelling the optimum grain size on the low cycle fatigue life of a $\mathrm{Ni}$ based superalloy in the presence of two possible crack initiation sites. Scr Mater 50:25-30. https://doi.org/10.1016/j.scriptamat.2003.09.043

45. Stinville JC, Martin E, Karadge M, Ismonov S, Soare M, Hanlon T, Sundaram S, Echlin MP, Callahan PG, Lenthe WC, Miller VMM, Miao J, Wessman AE, Finlay R, Loghin A, Marte J, Pollock TM (2018) Fatigue deformation in a polycrystalline nickel base superalloy at intermediate and high temperature: competing failure modes. Acta Mater 152:16-33. https://doi.org/10.1016/j. actamat.2018.03.035

46. Stinville J-CC, Martin E, Karadge M, Ismonov S, Soare M, Hanlon T, Sundaram S, Echlin MPLP, Callahan PG, Lenthe WC, Miao J, Wessman AE, Finlay R, Loghin A, Marte J, Pollock TM (2018) Competing modes for crack initiation from non-metallic inclusions and intrinsic microstructural features during fatigue in a polycrystalline nickel-based superalloy. Metall Mater Trans A 49:3865-3873. https://doi.org/10.1007/s11661-018-4780-3

47. Zhang T, Collins DM, Dunne FPE, Shollock BA (2014) Crystal plasticity and high-resolution electron backscatter diffraction analysis of full-field polycrystal Ni superalloy strains and rotations under thermal loading. Acta Mater 80:25-38. https://doi. org/10.1016/j.actamat.2014.07.036

48. Zhang T, Jiang J, Shollock BA, Ben BT, Dunne FPE (2015) Slip localization and fatigue crack nucleation near a non-metallic inclusion in polycrystalline nickel-based superalloy. Mater Sci Eng A 641:328-339. https://doi.org/10.1016/j.msea.2015.06.070

49. Zhang T, Jiang J, Britton B, Shollock B, Dunne F (2016) Crack nucleation using combined crystal plasticity modelling, highresolution digital image correlation and high-resolution electron backscatter diffraction in a superalloy containing non-metallic inclusions under fatigue. Proc R Soc A Math Phys Eng Sci 472:20150792. https://doi.org/10.1098/rspa.2015.0792

50. Naragani D, Sangid MD, Shade PA, Schuren JC, Sharma H, Park JS, Kenesei P, Bernier JV, Turner TJ, Parr I (2017) Investigation of fatigue crack initiation from a non-metallic inclusion via high energy x-ray diffraction microscopy. Acta Mater 137:71-84. https ://doi.org/10.1016/j.actamat.2017.07.027

51. Jiang J, Yang J, Zhang T, Dunne FPE, Ben BT (2015) On the mechanistic basis of fatigue crack nucleation in Ni superalloy containing inclusions using high resolution electron backscatter diffraction. Acta Mater 97:367-379. https://doi.org/10.1016/j. actamat.2015.06.035

52. Bandyopadhyay R, Sangid MD (2019) Crystal plasticity assessment of inclusion- and matrix-driven competing failure modes in a nickel-base superalloy. Acta Mater 177:20-34. https://doi. org/10.1016/j.actamat.2019.07.024

53. Texier D, Stinville J-C, Charpagne M-A, Chen Z, Valle V, Villechaise P, Pollock TM, Cormier J (2020) Role of non-metallic inclusions and twins on the variability in fatigue life in alloy 718 nickel base superalloy. In: Tin S, Hardy M, Clews J, Cormier J, Feng Q, Marcin J, O’Brien C, Suzuki A (eds) Superalloys 2020. Springer, Cham, pp 629-639

54. Parr IMD, Jackson TJ, Hardy MC, Child D, Argyrakis C, Severs K, Saraf V, Stumpf JM (2016) Inhomogeneous grain coarsening behavior in supersolvus heat treated nickel-based superalloy RR1000. In: Superalloys 2016. Wiley, Hoboken, pp 447-456

55. Mello AW, Nicolas A, Sangid MD (2017) Fatigue strain mapping via digital image correlation for Ni-based superalloys: the role of thermal activation on cube slip. Mater Sci Eng A 695:332-341. https://doi.org/10.1016/j.msea.2017.04.002

56. Wadell $\mathrm{H}$ (1935) Volume, shape and roundness of quartz particles. J Geol 43:250-280

57. Bandyopadhyay R, Prithivirajan V, Sangid MD (2019) Uncertainty quantification in the mechanical response of crystal plasticity 
simulations. JOM 71:2612-2624. https://doi.org/10.1007/s1183 7-019-03551-3

58. Prithivirajan V, Sangid MD (2018) The role of defects and critical pore size analysis in the fatigue response of additively manufactured IN718 via crystal plasticity. Mater Des 150:139-153. https ://doi.org/10.1016/j.matdes.2018.04.022

59. Yeratapally SR, Glavicic MG, Argyrakis C, Sangid MD (2017) Bayesian uncertainty quantification and propagation for validation of a microstructure sensitive model for prediction of fatigue crack initiation. Reliab Eng Syst Saf 164:110-123. https://doi. org/10.1016/j.ress.2017.03.006

60. Larsen JM, Jha SK, Szczepanski CJ, Caton MJ, John R, Rosenberger AH, Buchanan DJ, Golden PJ, Jira JR (2013) Reducing uncertainty in fatigue life limits of turbine engine alloys. Int J Fatigue 57:103-112. https://doi.org/10.1016/j.ijfat igue.2013.01.012

61. Luo C (2001) Modeling the behavior of inclusions in plastic deformation of steels. Royal Institute of Technology

62. Asmani M, Kermel C, Leriche A, Ourak M (2001) Influence of porosity on Youngs modulus and poisson's ratio in alumina ceramics. J Eur Ceram Soc 21:1081-1086. https://doi.org/10.1016/ S0955-2219(00)00314-9

63. Haggerty RP, Sarin P, Apostolov ZD, Driemeyer PE, Kriven WM (2014) Thermal expansion of $\mathrm{HfO} 2$ and $\mathrm{ZrO} 2$. J Am Ceram Soc 97:2213-2222. https://doi.org/10.1111/jace.12975

64. Grant BMB, Stone HJ, Withers PJ, Preuss M (2009) High-temperature strain field measurement using digital image correlation. J Strain Anal Eng Des 44:263-271. https://doi.org/10.1243/03093 247JSA478

65. Rolph J, Preuss M, Iqbal N, Hofmann M, Nikov S, Hardy MC, Glavicic MG, Ramanathan R, Evans A (2012) Residual stress evolution during manufacture of aerospace forgings. In: Superalloys 2012. Wiley, Hoboken, pp 881-891

66. Rice JR (1971) Inelastic constitutive relations for solids: an internal variables theory and its application to metal plasticity. J Mech Phys Solids 19:433-455. https://doi.org/10.1016/00225096(71)90010-X

67. Hutchinson JW (1976) Bounds and self-consistent estimates for creep of polycrystalline materials. Proc R Soc A Math Phys Eng Sci 348:101-127. https://doi.org/10.1098/rspa.1976.0027

68. Essmann U, Mughrabi H (1979) Annihilation of dislocations during tensile and cyclic deformation and limits of dislocation densities. Philos Mag A Phys Condens Matter Struct Defects Mech Prop 40:731-756. https://doi.org/10.1080/01418617908234871

69. Mecking H, Kocks UF (1981) Kinetics of flow and strain-hardening. Acta Metall 29:1865-1875. https://doi.org/10.1016/00016160(81)90112-7

70. Kocks UF, Mecking H (2003) Physics and phenomenology of strain hardening: the FCC case. Prog Mater Sci 48:171-273. https ://doi.org/10.1016/S0079-6425(02)00003-8

71. Kocks UF, Argon AS, Ashby MF (1975) Thermodynamics and kinetics of slip. Prog Mater Sci 19

72. Beyerlein IJ, Tomé CN (2008) A dislocation-based constitutive law for pure $\mathrm{Zr}$ including temperature effects. Int J Plast 24:867895. https://doi.org/10.1016/j.ijplas.2007.07.017

73. Frederick CO, Armstrong PJ (2007) A mathematical representation of the multiaxial Bauschinger effect. Mater High Temp 24:1-26. https://doi.org/10.1179/096034007X207589

74. McGinty RD (2001) Multiscale representation of polycrystalline inelasticity. Georgia Institute of Technology

75. Wang Y, Wang JJ, Zhang H, Manga VR, Shang SL, Chen L-Q, Liu Z-K (2010) A first-principles approach to finite temperature elastic constants. J Phys Condens Matter 22:225404. https://doi. org/10.1088/0953-8984/22/22/225404

76. Suresh S (1998) Fatigue of materials. Cambridge University Press, Cambridge
77. Cheng J, Shahba A, Ghosh S (2016) Stabilized tetrahedral elements for crystal plasticity finite element analysis overcoming volumetric locking. Comput Mech 57:733-753. https://doi. org/10.1007/s00466-016-1258-2

78. Parthasarathy VN, Graichen CM, Hathaway AF (1994) A comparison of tetrahedron quality measures. Finite Elem Anal Des 15:255-261. https://doi.org/10.1016/0168-874X(94)90033-7

79. Castelluccio GM, McDowell DL (2015) Microstructure and mesh sensitivities of mesoscale surrogate driving force measures for transgranular fatigue cracks in polycrystals. Mater Sci Eng A 639:626-639. https://doi.org/10.1016/J.MSEA.2015.05.048

80. Nicolas A, Co NEC, Burns JT, Sangid MD (2019) Predicting fatigue crack initiation from coupled microstructure and corrosion morphology effects. Eng Fract Mech 220:106661. https:// doi.org/10.1016/j.engfracmech.2019.106661

81. Rovinelli A, Sangid MD, Proudhon H, Ludwig W (2018) Using machine learning and a data-driven approach to identify the small fatigue crack driving force in polycrystalline materials. NPJ Comput Mater. https://doi.org/10.1038/s41524-018-0094-7

82. Rovinelli A, Sangid MD, Proudhon H, Guilhem Y, Lebensohn RA, Ludwig W (2018) Predicting the 3D fatigue crack growth rate of small cracks using multimodal data via Bayesian networks: Insitu experiments and crystal plasticity simulations. J Mech Phys Solids 115:208-229. https://doi.org/10.1016/j.jmps.2018.03.007

83. Smith RC (2013) Uncertainty quantification: theory, implementation, and applications. SIAM, Philadelphia

84. Metropolis N, Rosenbluth AW, Rosenbluth MN, Teller AH, Teller E (1953) Equation of state calculations by fast computing machines. J Chem Phys 21:1087-1092. https://doi. org/10.1063/1.1699114

85. Hastings WK (1970) Monte Carlo sampling methods using Markov chains and their applications. Biometrika 57:97-109

86. Cross R, Makeev A, Armanios E (2007) Simultaneous uncertainty quantification of fracture mechanics based life prediction model parameters. Int J Fatigue 29:1510-1515. https://doi.org/10.1016/j. ijfatigue.2006.10.027

87. Joseph DS, Chakraborty P, Ghosh S (2010) Wavelet transformation based multi-time scaling method for crystal plasticity FE simulations under cyclic loading. Comput Methods Appl Mech Eng 199:2177-2194. https://doi.org/10.1016/j.cma.2010.03.020

88. Chakraborty P, Joseph DS, Ghosh S (2011) Wavelet transformation based multi-time scale crystal plasticity FEM for cyclic deformation in titanium alloys under dwell load. Finite elements in analysis and design. Elsevier, Amsterdam, pp 610-618

89. Ghosh S, Chakraborty P (2013) Microstructure and load sensitive fatigue crack nucleation in Ti-6242 using accelerated crystal plasticity FEM simulations. Int J Fatigue 48:231-246. https://doi. org/10.1016/j.ijfatigue.2012.10.022

90. Chakraborty P, Ghosh S (2013) Accelerating cyclic plasticity simulations using an adaptive wavelet transformation based multitime scaling method. Int J Numer Methods Eng 93:1425-1454. https://doi.org/10.1002/nme.4459

91. Farooq H (2019) Crystal plasticity applied to aggregates under non-symmetric cyclic loadings: mechanical analysis and model order reduction. PSL Research University

92. Bin PY, Dunne FPE, MacLachlan DW (2020) A mechanistic and stochastic approach to fatigue crack nucleation in coarse grain RR1000 using local stored energy. Fatigue Fract Eng Mater Struct. https://doi.org/10.1111/ffe.13376

93. Gabb TP, Kantzos PT, Telesman J, Gayda J, Sudbrack CK, Palsa B (2011) Fatigue resistance of the grain size transition zone in a dual microstructure superalloy disk. Int J Fatigue 33:414-426. https://doi.org/10.1016/j.ijfatigue.2010.09.022 